\title{
Extended ALE Method for fluid-structure interaction problems with large structural displacements
}

\author{
Steffen Basting ${ }^{\mathrm{a}, *}$, Annalisa Quaini ${ }^{\mathrm{b}}$, Sunčica Čanić ${ }^{\mathrm{b}}$, Roland Glowinski $^{\mathrm{b}}$ \\ ${ }^{a}$ Institute of Applied Mathematics (LS III), TU Dortmund, Vogelpothsweg 8, 44227 Dortmund, Germany \\ ${ }^{b}$ Department of Mathematics, University of Houston, 4800 Calhoun Rd, Houston TX 77204, USA
}

\begin{abstract}
Standard Arbitrary Lagrangian-Eulerian (ALE) methods for the simulation of fluid-structure interaction (FSI) problems fail due to excessive mesh deformations when the structural displacement is large. We propose a method that successfully deals with this problem, keeping the same mesh connectivity while enforcing mesh alignment with the structure. The proposed Extended ALE Method relies on a variational mesh optimization technique, where mesh alignment with the structure is achieved via a constraint. This gives rise to a constrained optimization problem for mesh optimization, which is solved whenever the mesh quality deteriorates. The performance of the proposed Extended ALE Method is demonstrated on a series of numerical examples involving 2D FSI problems with large displacements. Two way coupling between the fluid and structure is considered in all the examples. The FSI problems are solved using either a DirichletNeumann algorithm, or a Robin-Neumann algorithm. The Dirichlet-Neumann algorithm is enhanced by an adaptive relaxation procedure based on Aitken's acceleration. We show that the proposed method has excellent performance in problems with large displacements, and that it agrees well with a standard ALE method in problems with mild displacement.
\end{abstract}

Keywords: Mesh optimization, Arbitrary Lagrangian-Eulerian formulation, Fluid-structure interaction, Domain decomposition methods.

\section{Introduction}

The focus of this work is on the numerical simulation of the motion of an elastic body immersed in an incompressible, viscous fluid, with the structure undergoing large displacements. A motivating example is the interaction between heart valves and blood flow. The difficulties associated with accurate numerical simulation of this class of fluid-structure interaction problems are: (1) large changes in the fluid domain that occur due to the large structural displacements; (2) accurate approximation of the hydrodynamic force at the fluid-structure interface; and (3) added mass effect, which is known to cause various numerical difficulties when the fluid and structure have comparable densities [76, 22]. Several numerical approaches have been proposed in the literature for this class of problems, each dealing with the above-mentioned difficulties in a different way.

To deal with the fluid domain motion associated with structural displacements, numerical methods can be classified into the methods with fixed meshes and the methods with moving meshes. The fixed mesh methods include the Immersed Boundary Method (IBM) [64, 66, 65], the Fictitious Domain Method [45, 44, 1], the level set method [27, 26, 42], the so called Eulerian FSI methods [31, 71, 81], and the deforming composite grids $[6,7,57]$. These methods rely on a fixed fluid mesh used in a fluid solver, while the presence of the structure is implemented in different ways. See also [72]. For example, in the Immersed Boundary Method

\footnotetext{
* Corresponding author

Email addresses: steffen.basting@math.tu-dortmund.de (Steffen Basting), quaini@math.uh.edu (Annalisa Quaini), canic@math.uh.edu (Sunčica Čanić), roland@math.uh.edu (Roland Glowinski)
} 
the fluid feels the structure through external forces acting on the fluid, where the coupling between the (fixed) fluid mesh and a (Lagrangian) structure mesh is performed via Dirac Delta functions. To get around the difficulties associated with the discretization of the Dirac Delta, and the low accuracy it causes in the calculation of the hydrodynamic force, modifications of the IBM were introduced. They include the extended Immersed Boundary Method [79], and the Immersed Finite Element Method [82]. On the other hand, in the Fictitious Domain Method, the coupling between the fluid and structure is enforced via Lagrange multipliers (imposing continuity of velocity, or the no-slip condition). This approach was applied first to problems with rigid particles and later to problems with flexible structures, where Lagrange multipliers were located along the structure surface $[3,50,77,78]$. In all the cases discussed above, adaptive mesh refinement typically needs to be used to obtain reasonable accuracy in the calculation of the hydrodynamic force acting on the structure. In the work presented in [57] the method of deforming composite grids was used to capture the motion of a beam modeled using a generalized Euler-Bernoulli beam model. An underlying fixed Cartesian mesh was used for the bulk of the fluid domain, with an overlapping grid consisting of a set of structured component grids to resolve the structure and boundary-fitted curvilinear grids at the structure boundary.

The moving mesh methods are typically based on Arbitrary Lagrangian-Eulerian (ALE) approaches, introduced in $[53,28]$ for FSI problems discretized with the Finite Element method. Earlier works [63, 51] introduced ALE methods for the Navier-Stokes equations in moving domains discretized with the Finite Difference method. Instead of being fixed, the fluid mesh follows the motion of the elastic body via a mapping, called the ALE mapping, which is calculated based on the current location of the structure (e.g., as a harmonic extension of the current interface position onto the fluid domain). ALE methods were proved to be accurate and robust for hemodynamics applications involving small mesh displacements (see, e.g., [35]). Although these methods offer many advantages provided by the explicit representation of the fluidstructure interface $[52,76,9]$, problems arise whenever strong deformations or even topological changes of the interface lead to a degeneration of the computational mesh. To deal with large structural displacements, remeshing was introduced in [30, 59, 58]. By "remeshing" it is normally meant that a new mesh with different connectivity is generated from scratch when the quality of the given mesh is poor.

Mixed ALE and fictitious domain formulations have also been proposed [49, 29]. These approaches also require adaptive mesh refinement for an accurate calculation of the viscous shear stresses on the solid boundary.

For completeness, we also mention a mesh-free Lattice-Boltzmann method [54, 34, 32, 21], which has also been used for the simulation of FSI problems with large structural displacements.

In the present work we propose a method which is a variant of an ALE approach, but it elegantly captures large structural displacements without changing mesh connectivity. The method retains the positive features of the ALE approaches, such as the accurate approximation of the fluid-structure interface and accurate representation of the hydrodynamic forces, without the need for adaptive mesh refinement. The method is based on a fixed "base" mesh that is adapted to approximate the interface via an ALE-type mapping, while maintaining mesh connectivity (nodes or elements are neither inserted nor removed). The fundamental building block is a variational mesh optimization approach that does not rely on any combinatorial considerations. Alignment of the optimized mesh with the structure interface is stated as a constraint of a mesh optimization problem thanks to a level set description of the geometry.

Our method can be regarded as an extension of the techniques introduced in $[80,12,13]$ for two-phase flows and one-way coupled FSI problems (i.e., the structure moves with a prescribed law). In contrast with the strategy proposed in $[80,12,13]$, in our approach the variational alignment procedure is only performed when the mesh becomes too distorted, making our methodology closer to a standard ALE approach, which is why we call it Extended ALE Method. The main features of the proposed Extended ALE approach are:

- Non-degenerate meshes of provably optimal quality;

- The alignment of the mesh with the interface, which allows for a simple definition and efficient implementation of problem-specific finite element spaces, such as the elements allowing discontinuities across the interface;

- Fixed mesh connectivity, which makes the method easy to implement in an existing standard ALE 
code.

Our approach is similar in spirit to the methods proposed in $[15,25,5]$ (the fixed-mesh ALE approach), and also to the methodology based on "universal meshes" (see, e.g. [70]) recently proposed in [41, 40], which all aim at providing discretization schemes defined on aligned meshes with fixed connectivity. In contrast to our optimization strategy, the mesh adaptation presented in [15] relies on explicit combinatorial considerations to create interface aligned meshes. Similarly, the methodology based on universal meshes presented in [41, 40] makes use of a "closest point projection" to create a discrete interface consisting of certain edges that have to be chosen based on combinatorial considerations. In [70] it is discussed under which conditions this leads to suitable meshes. On the other hand, the fixed mesh ALE approach virtually evolves the fluid mesh according to a standard ALE procedure, and then projects back the results on a fixed background mesh. However, cut elements have to be considered in order to enable an accurate imposition of boundary conditions. The main benefits of our variational approach are: no explicit combinatorial testing is needed and the resulting meshes are guaranteed to be non-degenerate.

To show that our approach has the desired features, we consider the interaction of an elastic beam with a 2D incompressible fluid. Although dealing with a simplified model, the problem under consideration retains important physical features common to more complex models: large displacement and added mass effect. We show that the Extended ALE Method allows to easily capture the pressure discontinuity across the interface, which coincides with the 1D elastic structure. Methods based on non-aligned fixed meshes cannot capture such a discontinuity, unless further techniques are used, such as, e.g., the enrichment of the function spaces as in X-FEM, see [39]. Moreover, thanks to the mesh alignment with the interface, the kinematic coupling condition is easily enforced.

Once a mesh has been obtained from the above mentioned constrained optimization problem, the FSI problem is solved with classical Domain Decomposition algorithms (see, e.g., [69]): either the DirichletNeumann method, which is combined with an Aitken's acceleration technique [55], or the Robin-Neumann method. It was shown in [4] that when the structure lies on part of the fluid domain boundary, the RobinNeumann method features excellent convergence properties: it always converges without any relaxation and its convergence is almost insensitive to the added-mass effect. Other schemes for FSI problems that use Robin interface conditions are presented in $[62,48,20,19,8,57]$. In particular, we mention [57] where a second-order accurate loosely-coupled scheme incorporating a Robin-type interface condition was used to study fluid-structure interaction between an incompressible, viscous fluid and a generalized Euler-Bernoulli beam. To the best of our knowledge, the Robin-Neumann method has never been applied to FSI problems involving an immersed structure.

The outline of the paper is as follows. In Section 2 we state the problem. The constrained optimization approach, which is at the core of our Extended ALE Method, is explained in Section 3. We describe the Domain Decomposition algorithms in Section 4, and summarize the numerical methods that we use for the time and space discretization of the fluid and structure problems in Section 5. In Section 6, we present numerical results obtained on a series of numerical tests carefully chosen to highlight the main features of the method. Conclusions are drawn in Section 7.

\section{Problem definition}

Consider a domain $\Omega \subset \mathbb{R}^{2}$ containing an elastic beam forming a 1D manifold $\Gamma(t) \subset \Omega$ whose location depends on time. The beam is surrounded by an incompressible, viscous fluid occupying domain $\Omega$, defining the time dependent fluid domain $\Omega_{f}(t):=\Omega \backslash \Gamma(t)$. The beam can be periodic (closed curve, see Fig. 1(a)) or non-periodic (open curve, see Fig. 1(b)).

\subsection{The fluid problem}

The fluid flow is governed by the Navier-Stokes equations for an incompressible, viscous fluid:

$$
\begin{array}{ll}
\rho_{f}\left(\frac{\partial \boldsymbol{u}}{\partial t}+(\boldsymbol{u} \cdot \nabla) \boldsymbol{u}\right)-\nabla \cdot \boldsymbol{\sigma}=\mathbf{0} & \text { in } \Omega_{f}(t), \\
\nabla \cdot \boldsymbol{u}=0 & \text { in } \Omega_{f}(t),
\end{array}
$$




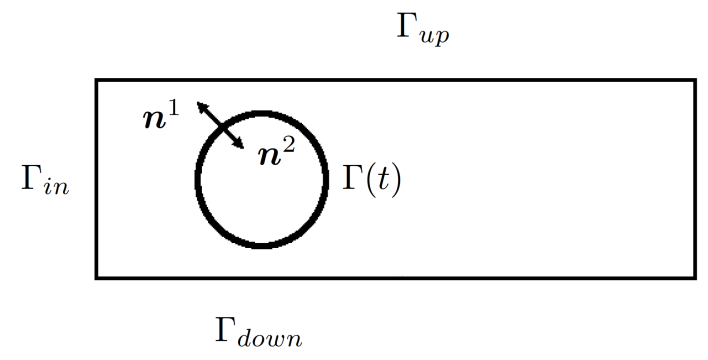

(a) Periodic beam

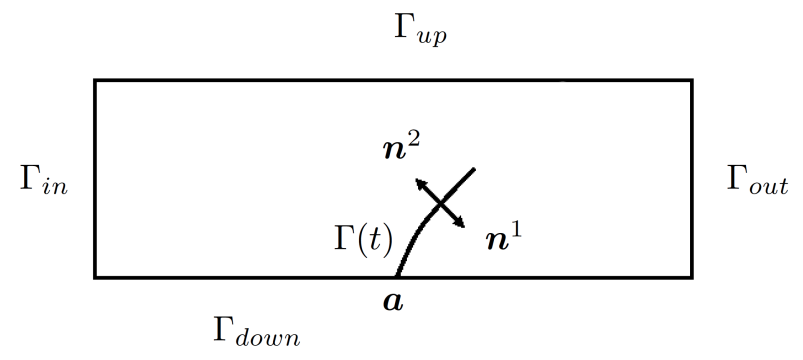

(b) Immersed beam

Figure 1: Computational domain for (a) the periodic beam case and (b) the immersed beam case.

for $t \in(0, T]$, where $\rho_{f}$ is the fluid density, $\boldsymbol{u}$ is the fluid velocity, and $\boldsymbol{\sigma}$ the Cauchy stress tensor. For Newtonian fluids $\boldsymbol{\sigma}$ has the following expression

$$
\boldsymbol{\sigma}(\boldsymbol{u}, p)=-p \mathbf{I}+2 \mu \boldsymbol{\epsilon}(\boldsymbol{u})
$$

where $p$ is the pressure, $\mu$ is the fluid dynamic viscosity, and $\boldsymbol{\epsilon}(\boldsymbol{u})=\left(\nabla \boldsymbol{u}+(\nabla \boldsymbol{u})^{T}\right) / 2$ is the strain rate tensor. Equations (1)-(2) need to be supplemented with initial and boundary conditions.

In order to describe the evolution of the fluid domain, we begin by adopting an Arbitrary LagrangianEulerian (ALE) approach [53]. More precisely, let $\hat{\Omega}_{f} \subset \mathbb{R}^{2}$ be a fixed reference domain. We consider a smooth ALE mapping

$$
\begin{aligned}
\mathcal{A} & : \quad[0, T] \times \hat{\Omega}_{f} \rightarrow \mathbb{R}^{2} \\
\mathcal{A}\left(t, \hat{\Omega}_{f}\right) & =\Omega_{f}(t), \quad \forall t \in[0, T] .
\end{aligned}
$$

For each time instant $t \in[0, T], \mathcal{A}$ is assumed to be a homeomorphism. The domain velocity $\mathbf{w}$ is defined as

$$
\mathbf{w}(t, \cdot)=\partial_{t} \mathcal{A}\left(t, \mathcal{A}(t, \cdot)^{-1}\right)
$$

For any sufficiently smooth function $F:[0, T] \times \mathbb{R}^{2} \rightarrow \mathbb{R}$, we may define the ALE time derivative of $F$ as

$$
\left.\frac{\partial F}{\partial t}\right|_{\hat{\mathbf{x}}}=\frac{\partial F}{\partial t}(t, \mathcal{A}(t, \hat{\mathbf{x}}))=\frac{\partial F}{\partial t}(t, \mathbf{x})+\mathbf{w}(t, \mathbf{x}) \cdot \nabla F(t, \mathbf{x})
$$

where $\mathbf{x}=\mathcal{A}(t, \hat{\mathbf{x}}), \hat{\mathbf{x}} \in \hat{\Omega}$. With these definitions, we can write the incompressible Navier-Stokes equations in ALE formulation as follows:

$$
\begin{array}{ll}
\left.\rho_{f} \frac{\partial \boldsymbol{u}}{\partial t}\right|_{\hat{\mathbf{x}}}+\rho_{f}(\boldsymbol{u}-\mathbf{w}) \cdot \nabla \boldsymbol{u}-\nabla \cdot \boldsymbol{\sigma}=\mathbf{0} & \text { in } \Omega_{f}(t), \\
\nabla \cdot \boldsymbol{u}=0 & \text { in } \Omega_{f}(t),
\end{array}
$$

for $t \in(0, T]$.

\subsection{The structure problem}

For the structure problem, we consider two structural models, both based on a linearly elastic beam equation:

- a periodic beam (PB) described by a linear beam equation, which results in balloon-type FSI problems;

- an inextensible beam (IB) giving rise to a non-linear problem, where the nonlinearity comes from the inextensibility condition. 
In both cases, we assume negligible torsional effects for the beam. Let us denote by $\rho_{s}$ the linear density (i.e. mass per unit length), by $L$ the length, and by $E I$ the flexural stiffness of the beam. We will use the following notation, with $s$ denoting arc length and $t$ time:

$$
\boldsymbol{y}^{\prime}=\frac{\partial \boldsymbol{y}}{\partial s}, \quad \dot{\boldsymbol{y}}=\frac{\partial \boldsymbol{y}}{\partial t}, \quad \boldsymbol{y}^{\prime \prime}=\frac{\partial^{2} \boldsymbol{y}}{\partial s^{2}}, \quad \ddot{\boldsymbol{y}}=\frac{\partial^{2} \boldsymbol{y}}{\partial t^{2}}
$$

\subsubsection{A periodic beam (PB) model}

Consider a periodic beam (closed curve). Its dynamic behavior is governed by the Euler-Lagrange equations:

$$
\rho_{s} \ddot{\boldsymbol{x}}+E I \boldsymbol{x}^{\prime \prime \prime \prime}=\boldsymbol{f} \quad \text { in }(0, L), t \in(0, T]
$$

where $\boldsymbol{x}=\boldsymbol{x}(t, s)$ is the parametric curve defining the beam position and $\boldsymbol{f}$ denotes the force acting on the beam. In our case, $f$ is the hydrodynamic force, which will be specified in Sec. 2.3. Problems similar to (5) were considered, for instance, in [23, 24] and the references therein. Equation (5) has to be supplemented with initial and boundary conditions. We enforce periodic boundary conditions:

$$
\boldsymbol{x}(0)=\boldsymbol{x}(L), \quad \boldsymbol{x}^{\prime}(0)=\boldsymbol{x}^{\prime}(L) .
$$

\subsubsection{An inextensible beam (IB) model}

In the second case, we consider a non-periodic (open curve), inextensible beam [46, 43, 29]. The structural model is based on equation (5) with an additional constraint of inextensibility, i.e. the beam cannot shrink or stretch during its interaction with the fluid. The resulting structure problem is non-linear, and the numerical treatment of this problem is much more challenging than the problem described in Sec. 2.2.1.

Using the virtual work principle, the beam motion for $t \in(0, T]$ is modeled by the following. Find $\boldsymbol{x}(t) \in K$ such that:

$$
\int_{0}^{L} \rho_{s} \ddot{\boldsymbol{x}} \cdot \boldsymbol{y} d s+\int_{0}^{L} E I \boldsymbol{x}^{\prime \prime} \cdot \boldsymbol{y}^{\prime \prime} d s=\int_{0}^{L} \boldsymbol{f} \cdot \boldsymbol{y} d s, \quad \forall \boldsymbol{y} \in d K(\boldsymbol{x}),
$$

with

$$
\begin{aligned}
K & =\left\{\boldsymbol{y} \in\left(H^{2}(0, L)\right)^{2},\left|\boldsymbol{y}^{\prime}\right|=1, \boldsymbol{y}(0)=\boldsymbol{a}, \boldsymbol{y}^{\prime}(0)=\boldsymbol{b}\right\}, \\
d K(\boldsymbol{x}) & =\left\{\boldsymbol{y} \in\left(H^{2}(0, L)\right)^{2}, \boldsymbol{x}^{\prime} \cdot \boldsymbol{y}^{\prime}=0, \boldsymbol{y}(0)=\mathbf{0}, \boldsymbol{y}^{\prime}(0)=\mathbf{0}\right\},
\end{aligned}
$$

and boundary conditions

$$
\boldsymbol{x}(0)=\boldsymbol{a}, \quad \boldsymbol{x}^{\prime}(0)=\boldsymbol{b}, \quad \boldsymbol{x}^{\prime \prime}(L)=\boldsymbol{x}^{\prime \prime \prime}(L)=0 .
$$

The conditions at $s=0$ are called the essential boundary conditions, describing a clamped beam, while the conditions at $s=L$ are called the natural boundary conditions. The non-linear inextensibility condition for the beam, $\left|\boldsymbol{x}^{\prime}\right|=1$, is embedded into the set $K$.

\subsection{The coupled fluid-structure interaction problem}

We consider two-way coupling between the fluid and structure: the motion of the beam is driven by the contact force exerted by the fluid, while at the same time the motion of the beam influences the fluid motion. The coupling conditions are described by the following. Let us denote the interface by

$$
\Gamma(t)=\{\boldsymbol{x}(t, s), s \in[0, L]\} .
$$

Let $\boldsymbol{n}^{1}$ be the unit normal vector pointing to the "left" (left with respect to the parameterization of $\boldsymbol{x}$ ) and $\boldsymbol{n}^{2}=-\boldsymbol{n}^{1}$ is the unit normal pointing to the "right", see Figure 1. Notice that the fluid-structure interface 
coincides with the structure domain. The hydrodynamic force acting on the structure (beam) is given by the jump in the normal fluid stress across the interface $\Gamma(t)$ :

$$
\boldsymbol{f}_{\Gamma}=-\boldsymbol{\sigma}^{1} \boldsymbol{n}^{1}-\boldsymbol{\sigma}^{2} \boldsymbol{n}^{2}
$$

where $\boldsymbol{\sigma}^{i}(\mathbf{x})=\lim _{\epsilon \rightarrow 0^{-}} \boldsymbol{\sigma}\left(\mathbf{x}+\epsilon \boldsymbol{n}^{i}\right), \mathbf{x} \in \Gamma, i=1,2$. Using this notation, we can now state the coupling conditions. For $t \in(0, T]$, the fluid problem $(3),(4)$ and the structure problems $(\mathbf{P B})$ or $(\mathbf{I B})$ are coupled by the following two conditions:

1. kinematic coupling condition (continuity of velocity, i.e., the no-slip condition)

$$
\boldsymbol{u}=\dot{\boldsymbol{x}} \quad \text { on } \Gamma(t)
$$

2. dynamic coupling condition (balance of contact forces)

$$
\boldsymbol{f}_{\Gamma}=\boldsymbol{f} \quad \text { on } \Gamma(t),
$$

where $\boldsymbol{f}$ is given by Eq. (5) for problem (PB), or by Eq. (7) for problem (IB).

Here, notation $\boldsymbol{u}=\dot{\boldsymbol{x}}$ in (12) is used to express the relation $\boldsymbol{u}(t, \boldsymbol{x}(t, s))=\dot{\boldsymbol{x}}(t, s), s \in[0, L]$ (analogously for $\boldsymbol{f}_{\Gamma}$ and $\boldsymbol{f}$ in (13)). Since $\boldsymbol{x}$ denotes the location of structure points and not the structure displacement, both the structure and fluid are given in Eulerian coordinates.

For the purposes that will be clear below when we introduce the Robin-Neumann scheme, we remark here that the coupling conditions (12)-(13) can be written in an equivalent form by introducing the constants $\alpha_{f}>0$ and $\alpha_{s}>0\left(\alpha_{f} \neq \alpha_{s}\right)$, and writing:

$$
\begin{array}{cc}
\alpha_{f} \boldsymbol{u}-\boldsymbol{f}_{\Gamma}=\alpha_{f} \dot{\boldsymbol{x}}-\boldsymbol{f} & \text { on } \Gamma(t), \\
\alpha_{s} \boldsymbol{u}+\boldsymbol{f}=\alpha_{s} \dot{\boldsymbol{x}}+\boldsymbol{f}_{\Gamma} & \text { on } \Gamma(t) .
\end{array}
$$

\section{Numerical Representation of the Geometry}

The main feature of our Extended ALE method is a variational mesh optimization technique combined with an additional constraint to enforce the alignment of the structure interface with the edges of the resulting triangulation. The mesh optimization, explained in Sec. 3.1 together with the alignment constraint described in Sec. 3.2 corresponds to a reparametrization of the ALE mapping.

\subsection{Optimal triangulations}

Let $\mathcal{T}$ be an initial triangulation of the domain $\Omega$ (not necessarily approximating the structure interface at this stage). Following a variational mesh optimization technique introduced by M. Rumpf in [73], we aim at finding an "optimal" triangulation $\mathcal{T}_{\text {opt }}$ resulting from an optimal mesh deformation $\chi_{\text {opt }}$ of $\mathcal{T}$, i.e. $\mathcal{T}_{\text {opt }}=\chi_{\text {opt }}(\mathcal{T})$. Deformation $\chi_{\text {opt }}$ belongs to the set $D$ of piecewise affine, orientation preserving, and globally continuous deformations:

$$
D=\left\{\chi \in\left(\mathcal{C}^{0}(\Omega)\right)^{2}:\left.\nabla \chi\right|_{T} \in \mathrm{GL}(2), \operatorname{det}\left(\left.\nabla \chi\right|_{T}\right)>0, \forall T \in \mathcal{T}\right\},
$$

with $\mathrm{GL}(2)=\left\{A \in \mathbb{R}^{2 \times 2}: \operatorname{det}(A) \neq 0\right\}$.

Deformation $\chi_{\mathrm{opt}} \in D$ is "optimal" in the sense that it is the argument for which a certain functional $\mathcal{F}$ attains its minimum value:

$$
\mathcal{F}\left(\chi_{\mathrm{opt}}\right)=\min _{\chi \in D} \mathcal{F}(\chi)
$$

We assume that the functional in (16) can be represented by a sum of weighted, element-wise contributions $F_{T}$ :

$$
\mathcal{F}(\chi)=\sum_{T \in \mathcal{T}} \mu_{T} F_{T}(\chi)
$$


where $\mu_{T}>0$ denotes a positive weight with $\sum_{T} \mu_{T}=1$. For each element $T \in \mathcal{T}$, let $R_{T}$ denote the linear reference mapping from a prescribed reference element $T_{\text {opt }}$ (an equilateral simplex with customizable edge length $h$ ) to $T$. We also define $R_{T}(\chi):=\chi \circ R_{T}$ for a given deformation $\chi$. The idea is to find mappings $\chi$ that would map the element $T$ onto the reference simplex $T_{\mathrm{opt}}$, i.e. such that the optimal deformation would fulfill $\chi_{\mathrm{opt}}=R_{T}^{-1}$.

Under the assumptions of translational invariance, isotropy and frame indifference of the functionals, it can be shown (see [73]) that in two dimensions $F_{T}$ may be expressed as a function of the invariants $\left\|\nabla R_{T}(\chi)\right\|^{2}$ and $\operatorname{det}\left(\nabla R_{T}(\chi)\right)$. For example, given a function $\tilde{F}_{T}: \mathbb{R} \times \mathbb{R} \rightarrow \mathbb{R}$ we can write:

$$
F_{T}(\chi)=\tilde{F}_{T}(a, d):=\tilde{F}_{T}\left(\left\|\nabla R_{T}(\chi)\right\|^{2}, \operatorname{det}\left(\nabla R_{T}(\chi)\right)\right) .
$$

Here, $\|\cdot\|$ denotes the Frobenius norm. Note that the quantity $\left\|\nabla R_{T}(\chi)\right\|^{2}$ measures the change of edge lengths with respect to the reference element, and $\operatorname{det}\left(\nabla R_{T}(\chi)\right)$ measures the change in area.

In order to rule out deformations with vanishing determinant, we need

$$
\lim _{\operatorname{det}\left(\nabla R_{T}(\chi)\right) \rightarrow 0} F_{T}(\chi)=\infty
$$

With the additional assumption that the local functional $F_{T}(\chi)=\tilde{F}_{T}(a, d)$ is polyconvex (i.e. $\tilde{F}_{T}(a, d)$ is convex with respect to each argument), it can be proven that an optimal deformation exists and is globally injective [73].

A classical example of such a function $F_{T}$ is given by

$$
F_{T}(\chi)=\left(\left\|\nabla R_{T}(\chi)\right\|^{2}-2\right)^{2}+\operatorname{det}\left(\nabla R_{T}(\chi)\right)+\frac{1}{\operatorname{det}\left(\nabla R_{T}(\chi)\right)} .
$$

Notice that the optimally deformed simplex is obtained if $\left.\chi_{\mathrm{opt}}\right|_{T}=R_{T}^{-1}$, i.e. if

$$
F_{T}\left(\chi_{\mathrm{opt}}\right)=\tilde{F}_{T}\left(\|\mathbf{I}\|^{2}, \operatorname{det}(\mathbf{I})\right)=(2-2)^{2}+1+1=2 .
$$

We would also like to point out that the mesh optimization procedures proposed by Freitag and Knupp in e.g. [37, 38] which are based on minimizing the condition number of the mapping $R_{T}(\chi)$ are in the same spirit as the approach pursued here.

The variational mesh smoothing approach described above has several advantages:

- Minimization problem (16) yields triangulations which are provably optimal in the sense of the local measure (17).

- These triangulations can be shown to be non-degenerate, i.e. no self-intersection of elements occurs. This is the main property needed by our optimization approach.

- The element-wise representation of $\mathcal{F}$ provides built-in, local mesh quality control.

The price to pay for those advantages is that functional $\mathcal{F}$ in (16) is highly non-linear, non-convex, and global minimizers may not be unique.

\subsection{Interface aligned mesh}

We are now interested in having a triangulation that is non-degenerate, optimal (as explained in the previous subsection) and aligned with the beam position $\Gamma(t)$, i.e. we want the optimal triangulation edges to approximate $\Gamma(t)$. For this purpose, we introduce the following auxiliary tools:

- a "tubular box" around the structure of width $\delta$, denoted by $\Omega_{f}^{\delta}(t) \subset \Omega_{f}(t)$, within which mesh optimization with alignment will be performed, see Fig. 2(a); 


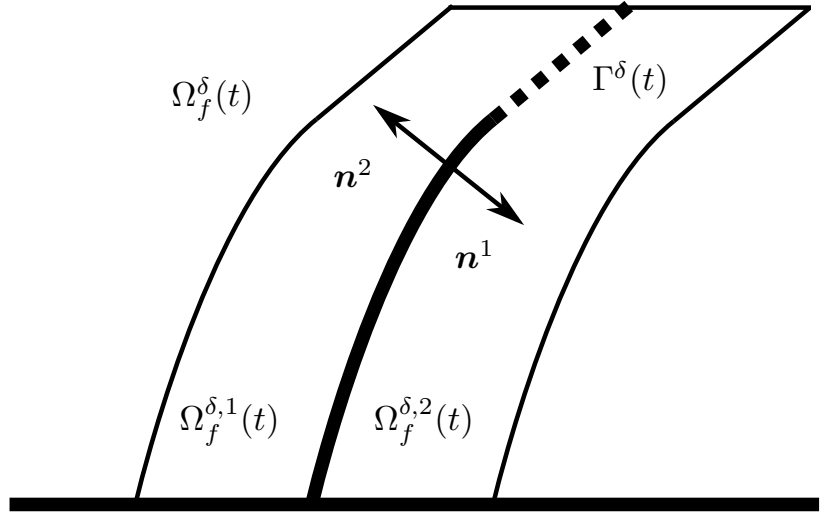

(a) Tubular box $\Omega_{f}^{\delta}(t)$

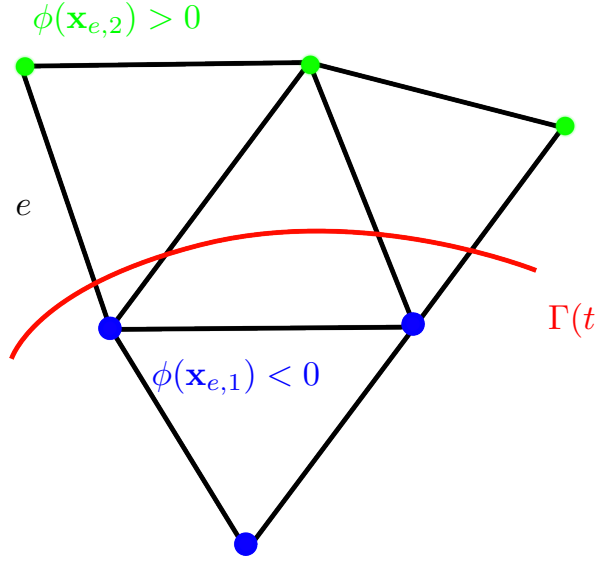

(b) Fluid mesh intersected by $\Gamma(t)$

Figure 2: (a) Tubular box $\Omega_{f}^{\delta}(t)$ around the structure position $\boldsymbol{x}$, zoomed in view of Fig. 1(b), and (b) $\Gamma(t)$ intersecting elements of the fluid mesh.

- a continuous level set function $\phi:[0, T] \times \Omega_{f}^{\delta}(t) \rightarrow \mathbb{R}$ whose zero level set includes the structure position $\boldsymbol{x}$ :

$$
\left.\begin{array}{rl}
\Omega_{f}^{\delta, 1}(t) & =\left\{\boldsymbol{y} \in \Omega_{f}^{\delta}(t): \phi(t, \boldsymbol{y})>0\right. \\
\Omega_{f}^{\delta, 2}(t) & =\left\{\boldsymbol{y} \in \Omega_{f}^{\delta}(t): \phi(t, \boldsymbol{y})<0\right. \\
\Gamma^{\delta}(t) & =\left\{\boldsymbol{y} \in \Omega_{f}^{\delta}(t): \phi(t, \boldsymbol{y})=0\right.
\end{array}\right\},
$$

where $\Gamma^{\delta}(t)$ denotes a "natural" extension of $\Gamma(t)$ to the boundary of $\Omega_{f}^{\delta}(t)$, and $\Omega_{f}^{\delta, 1}(t)$ and $\Omega_{f}^{\delta, 2}(t)$ denote the fluid sub-domains located on the "left" and "right" side of $\Gamma^{\delta}(t)$, respectively. See Fig. $2(\mathrm{a})$. Notice that $\boldsymbol{n}^{1}$ (resp., $\boldsymbol{n}^{2}$ ) is the outward unit normal on $\Gamma^{\delta}(t)$ of $\Omega_{f}^{\delta, 1}(t)$ (resp., $\left.\Omega_{f}^{\delta, 2}(t)\right)$.

Within $\Omega_{f}^{\delta}(t)$ we perform the following procedure. Let $e$ be an arbitrary edge of the triangulation $\mathcal{T}$ intersected by $\Gamma^{\delta}(t)$ as shown in Fig. 2(b), and let $\mathbf{x}_{e, 1}$ and $\mathbf{x}_{e, 2}$ be its endpoints. Due to continuity of $\phi$ and assumption (18), we observe that

$$
\phi\left(\mathbf{x}_{e, 1}\right) \phi\left(\mathbf{x}_{e, 2}\right)<0
$$

if and only if $e$ is intersected by $\Gamma^{\delta}(t)$, provided that the mesh size $h$ is sufficiently small to resolve the shape of $\Gamma^{\delta}(t)$. We therefore define the triangulation to be linearly aligned with $\Gamma(t)$ if

$$
\phi\left(\mathbf{x}_{e, 1}\right) \phi\left(\mathbf{x}_{e, 2}\right) \geq 0 \text { for all } e \in \mathcal{T} \text {. }
$$

We introduce a scalar constraint $c: D \rightarrow \mathbb{R}_{0}^{+}$, defined on $D$ given by (15), such that:

$$
c(\chi)=\sum_{e \in \chi(\mathcal{T})} \mathcal{H}\left(\phi\left(\mathbf{x}_{e, 1}\right) \phi\left(\mathbf{x}_{e, 2}\right)\right) \quad \text { where } \quad \mathcal{H}(z)= \begin{cases}>0 & \text { if } z<0 \\ =0 & \text { otherwise }\end{cases}
$$

Only deformations $\chi$ for which $c(\chi)=0$ will give aligned triangulations. Thus, a linearly aligned triangulation of optimal quality is obtained from the following constrained minimization problem:

$$
\min _{\chi \in D} \mathcal{F}(\chi) \text { such that } c(\chi)=0
$$


Given an aligned triangulation $\mathcal{T}$, we may define a linear approximation of the interface as

$$
\Gamma_{h}=\left\{\text { edges } e \in \mathcal{T}: \phi\left(\mathbf{x}_{e, i}\right)=0 \text { and } \mathbf{x}_{e, i} \in \Gamma \text { for } i=1,2\right\}
$$

In order to obtain a more accurate representation of the structure, we also consider piecewise quadratic approximations of $\Gamma(t)$. We make use of quadratic isoparametric finite elements equipped with additional degrees of freedom located at the edges.

\subsection{Details on the implementation}

In this subsection we provide additional details on our numerical realization of the minimization problem (19). One of the difficulties is how to handle the condition $\operatorname{det}\left(\left.\nabla \chi\right|_{T}\right)>0$ for admissible deformations $\chi \in D$. In practice, it is convenient to work with a standard finite element space such as

$$
X:=\left\{\chi \in\left(\mathcal{C}^{0}(\Omega)\right)^{2}:\left.\chi\right|_{T} \in\left(\mathbb{P}^{1}(T)\right)^{2} \forall T \in \mathcal{T}\right\}
$$

which consists of vector-valued, piecewise linear functions. Notice that $D \subset X$. In order to guarantee that the condition $\operatorname{det}\left(\left.\nabla \chi\right|_{T}\right)>0$ holds true for $\chi \in X$, we replace the local functional $F_{T}(\chi)$ in Eq. (17) by

$$
F_{T}(\chi)=(a-2)^{2}+d+\frac{\tilde{C}(\varepsilon)}{\varepsilon+d+|d|},
$$

where again $a=\left\|\nabla R_{T}(\chi)\right\|^{2}, d=\operatorname{det}\left(\nabla R_{T}(\chi)\right)$, and $0<\varepsilon \ll 1$ denotes a regularization parameter to avoid division by zero. For the numerical results in Sec. 6 we set $\epsilon=10^{-8}$. The constant $\tilde{C}(\varepsilon)=\frac{1}{2}(2+\varepsilon)^{2}$ guarantees that $d+\frac{\tilde{C}(\varepsilon)}{\varepsilon+d+|d|}$ assumes its minimum value for $d=1$. We remark that $F_{T}$ blows up for $d<0$ but coincides with the original contribution (17) for $d>0$ (up to regularization). Further details on the numerical implementation together with an evaluation of the mesh, approximation quality, and computational costs can be found in $[12,10,80]$.

In the following, for given a structure position $\Gamma(t)$, we will denote the optimal interface aligned triangulation obtained from the strategy outlined above by $\mathcal{T}_{\text {opt }}(\Gamma(t))$. The resulting computational domain is given by:

$$
\Omega_{f, \mathrm{opt}}(t)=\bigcup_{K \in \mathcal{T}_{\mathrm{opt}}(\Gamma(t))} K
$$

\section{Partitioned methods for the fluid-structure interaction problem}

The FSI problems described in Sec. 2 will be solved using two different partitioned strategies based on Domain Decomposition methods [69]: the Dirichlet-Neumann (DN) and the Robin-Neumann (RN) algorithms. Partitioned method are appealing for solving multi-physics problems such as those discussed in this manuscript, because they allow the reuse of existing fluid and structure solvers with minimal modifications. Because of the modularity of DN and RN algorithms, each physics sub-problem is solved separately, with the coupling conditions enforced in an iterative fashion. In the DN algorithm the coupling boundary condition (12) is imposed at the interface as a Dirichlet boundary condition for the fluid sub-problem, whereas in the $\mathrm{RN}$ algorithm the fluid sub-problem is endowed with Robin interface condition (14). In both algorithms, the structure sub-problem is supplemented with the Neumann "boundary condition" (13). Eq. (13) is a proper Neumann boundary condition when the structure is thick; for thin structures Eq. (13) prescribes a load on the structure.

To describe the DN and RN algorithms, we introduce the time-discretization step $\Delta t>0$ and set $t^{n}=n \Delta t$, for $n=1, \ldots, N$, with $N=T / \Delta t$. At every time $t^{n}$, the DN and RN algorithms iterate over the fluid and structure sub-problems until convergence. These are Richardson (also called fixed point) iterations for the position of $\Gamma\left(t^{n}\right)$. Let $k$ be the index for these iterations. 


\subsection{The Dirichlet-Neumann method}

At time $t^{n+1}$, iteration $k+1$, assuming that $\Omega_{f}^{n}, \boldsymbol{u}_{k}, p_{k}$, and $\boldsymbol{x}_{k}$ are known, the following steps are performed:

- Step 1: Solve the fluid sub-problem for the flow variables $\boldsymbol{u}_{k+1}, p_{k+1}$ defined on $\Omega_{f}^{n}$, with Dirichlet boundary condition

$$
\boldsymbol{u}_{k+1}=\dot{\boldsymbol{x}}_{k} \quad \text { on } \quad \Gamma^{n}
$$

- Step 2: Solve the structure sub-problem for the structure position $\boldsymbol{x}_{k+1}$, driven by the just calculated hydrodynamic force $\boldsymbol{f}_{\Gamma, k+1}$, i.e., $\boldsymbol{f}_{k+1}=\boldsymbol{f}_{\Gamma, k+1}$ on $\Gamma^{n}$.

- Step 3: Check the stopping criterion, e.g.

$$
\frac{\left\|\boldsymbol{x}_{k+1}-\boldsymbol{x}_{k}\right\|}{\left\|\boldsymbol{x}_{k}\right\|}<\epsilon,
$$

where $\epsilon$ is a given stopping tolerance. If violated, repeat Steps $1-3$. If satisfied, set $\boldsymbol{x}^{n+1}=\boldsymbol{x}_{k+1}$ and $p^{n+1}=p_{k+1}$.

- Step 4: Check the mesh quality of $\Omega_{f}^{n}$ :

- If good: Accept and set $\tilde{\boldsymbol{u}}^{n+1}=\boldsymbol{u}_{k+1}$ and $\tilde{\Omega}_{f}^{n+1}=\Omega_{f}^{n}$.

- If bad: Apply mesh optimization to get $\Omega_{f, \text { opt }}^{n}$, set $\tilde{\Omega}_{f}^{n+1}=\Omega_{f, \text { opt }}^{n}$. Project data onto new mesh, i.e.

$$
\tilde{\boldsymbol{u}}^{n+1}=I_{\Omega_{f}^{n} \rightarrow \tilde{\Omega}_{f}^{n+1}}\left(\boldsymbol{u}_{k+1}\right) .
$$

A mesh is considered to be "bad" if the maximum angle of the elements exceeds a certain value, for instance 130 degrees.

- Step 5: Standard ALE update: From the new structure position $\boldsymbol{x}^{n+1}$ obtain $\Gamma^{n+1}$, and from the intermediate fluid domain $\tilde{\Omega}_{f}^{n+1}$ obtain:

$$
\Omega_{f}^{n+1}=E\left(\Gamma^{n+1}, \tilde{\Omega}_{f}^{n+1}\right)
$$

using an extension operator $E$ (see comment below). Set $\boldsymbol{u}^{n+1}=\tilde{\boldsymbol{u}}^{n+1}$ and move to the next time step.

Some remarks on the scheme outlined above are in order:

1. For our computations, we do not use a "standard" extension operator $E$ (such as harmonic extension, or operators stemming from linear elasticity), but use the variational approach based on (16), (17). In our experience, this approach is superior to linear extension operators in terms of mesh quality.

2. The "inner" loop, in which the index $k$ changes, corresponds to Steps 1-3 in the above iteration algorithm. In those steps, the fluid domain is "frozen", which allows for important saving of computational time.

3. The angle-based criterion used to detect "bad" meshes in Step 4 is purely heuristic and may be replaced by other meaningful mesh quality criteria. The criterion should be sufficiently mild in order to prevent the reparametrization at every time step. Notice that the mesh optimization procedure presented in Subsec. 3.1 aims at generating triangulations made of equilateral triangles, therefore the angles after optimization are usually bounded away from, e.g., 130 degrees. 
4. A crucial point in the above algorithm is the choice of the mesh transfer operator $I_{\tilde{\Omega}_{f, n+1} \rightarrow \Omega_{f, \mathrm{opt}}^{n+1}}$ appearing in Eq. (22) at Step 4, needed whenever reparameterization is performed. In our case, this operator is the Lagrange interpolation operator which was also proposed in [40]. However, it is known that dynamically changing meshes may lead to spurious oscillations of the pressure for small time step sizes [16, 18]. Indeed, we will observe those oscillations in our numerical results, as shown in Section 6.5.2. Transferring the solution from one mesh to another without introducing these errors seems to be an open question.

It is well known that the convergence properties of the DN algorithm depend heavily on the added-mass effect [22]. In fact, when the structure constitutes a part of the fluid domain boundary, the number of DN iterations required to satisfy the stopping criterion (21) increases as the structure density approaches the fluid density. Moreover, below a certain density ratio $\rho_{s} / \rho_{f}$, which depends on the domain geometry, relaxation is needed for the DN algorithm to converge [60,61, 22]. This is why we adopt a simple Aitken's acceleration technique, which is based on a relaxation approach, and is known to reduce the number of DN iterations. This strategy, introduced in [55], was proposed for a setting similar to ours in [2]. The results in [2] indicate that only a few accelerated DN sub-iterations are to be expected for FSI problems with an immersed structure and large added-mass effect.

Dirichlet-Neumann algorithms, however, have been shown in [4] to fail for FSI problems with ballon-type structures such as the periodic beam case (PB). This is because in the DN algorithm the coupling conditions are satisfied asynchronously. As a result, the fluid sub-problem uses Dirichlet boundary condition $\boldsymbol{u}_{k+1}=\dot{\boldsymbol{x}}_{k}$ which is based on the velocity of the structure $\dot{\boldsymbol{x}}_{k}$ calculated from the previous sub-iteration. This $\dot{\boldsymbol{x}}_{k}$ may not be consistent with the incompressibility condition (2), which requires that

$$
0=\int_{\Gamma^{n}} \boldsymbol{u}_{k+1} \cdot \boldsymbol{n} \mathrm{d} \Gamma^{n}=\int_{\Gamma^{n}} \dot{\boldsymbol{x}}_{k} \cdot \boldsymbol{n} \mathrm{d} \Gamma^{n}
$$

Since the last integral is not necessarily equal to zero for all $\dot{\boldsymbol{x}}_{k}$, the fluid sub-problem in Step 1 is solved with a velocity prescribed at the boundary that does not satisfy the integral equality above, giving rise to an ill-posed problem at the semi-descrete level. Because of this limitation, in the next subsection we consider a Robin-Neumann algorithm for the solution of the FSI problem involving the periodic beam case (PB).

\subsection{The Robin-Neumann method}

At time $t^{n+1}$, iteration $k+1$, the following steps are performed:

- Step 1: Solve the fluid sub-problem for the flow variables $\boldsymbol{u}_{k+1}, p_{k+1}$ defined on $\Omega_{f}^{n}$, with Robin boundary condition

$$
\alpha_{f} \boldsymbol{u}_{k+1}-\boldsymbol{f}_{\Gamma, k+1}=\alpha_{f} \dot{\boldsymbol{x}}_{k}-\boldsymbol{f}_{k} \quad \text { on } \quad \Gamma^{n} .
$$

- Step 2, 3, 4 and 5 as in Sec. 4.1

Recall that $\boldsymbol{f}_{\Gamma}$ denotes the jump in the normal stress across the structure, as defined in (11), and $\boldsymbol{f}$ stands for the right hand-side of the structure equation (5). Notice that the DN algorithm can be interpreted as a particular case of the RN algorithm for $\alpha_{f} \rightarrow \infty$.

It was shown in [4] that when the structure constitutes a part of the fluid domain boundary for a suitable choice of parameter $\alpha_{f}$ the RN method features excellent convergence properties: it always converges without any relaxation and its convergence is insensitive to the added-mass effect. To our knowledge, the RN method has never been applied to FSI problems involving an immersed structure and a complex structure model like (PB) or (IB). Our goals are: to check whether these improved convergence properties still hold when the structure is immersed in the fluid domain, and to show that the RN algorithm can handle balloon-type FSI problems. 
In [4], $\alpha_{f}$ is estimated by considering a simplified structure model. Here, we follow the same approach. Under the hypothesis that the term $E I \boldsymbol{x}^{\prime \prime \prime \prime \prime}$ (as well as the inextensibility condition when problem IB is considered) is negligible, the structure model (5) reduces to a simple inertial model:

$$
\rho_{s} \ddot{\boldsymbol{x}}=\boldsymbol{f}_{\Gamma}, \quad \text { in }(0, L), t \in(0, T] .
$$

By discretizing this equation in time with the implicit Euler scheme (see, e.g., [68]) and by using the kinematic coupling condition (12), at the time $t^{n+1}$ we obtain:

$$
\frac{\rho_{s}}{\Delta t} \boldsymbol{u}^{n+1}-\boldsymbol{f}_{\Gamma}^{n+1}=\frac{\rho_{s}}{\Delta t^{2}}\left(\boldsymbol{x}^{n}-\boldsymbol{x}^{n-1}\right),
$$

which is a Robin boundary condition for the fluid problem. Thus, when a simple inertial model for the structure is adopted, the structure problem is not solved separately, but it is embedded in the fluid problem in the form of the Robin boundary condition (25). This simplified problem motivates the choice for the constant $\alpha_{f}$ in (24):

$$
\alpha_{f}=\frac{\rho_{s}}{\Delta t}
$$

for general structure models.

By plugging (26) into Robin condition (24) and taking into account Eq. (5) at iteration $k$, we get:

$$
\frac{\rho_{s}}{\Delta t} \boldsymbol{u}_{k+1}-\boldsymbol{f}_{\Gamma, k+1}=\frac{\rho_{s}}{\Delta t} \dot{\boldsymbol{x}}_{k}-(\underbrace{\rho_{s} \ddot{\boldsymbol{x}}_{k}+E I \boldsymbol{x}_{k}^{\prime \prime \prime \prime}}_{\boldsymbol{f}_{k}}),
$$

This equation is discretized in time with the implicit Euler scheme to obtain:

$$
\frac{\rho_{s}}{\Delta t} \boldsymbol{u}_{k+1}-\boldsymbol{f}_{\Gamma, k+1}=\frac{\rho_{s}}{\Delta t} \dot{\boldsymbol{x}}_{k}-\rho_{s} \frac{\dot{\boldsymbol{x}}_{k}-\dot{\boldsymbol{x}}^{n}}{\Delta t}-E I \boldsymbol{x}_{k}{ }^{\prime \prime \prime \prime} .
$$

The two terms containing $\dot{\boldsymbol{x}}_{k}$ cancel out, and a further discretization of $\dot{\boldsymbol{x}}^{n}$ leads to:

$$
\frac{\rho_{s}}{\Delta t} \boldsymbol{u}_{k+1}-\boldsymbol{f}_{\Gamma, k+1}=\frac{\rho_{s}}{\Delta t^{2}}\left(\boldsymbol{x}^{n}-\boldsymbol{x}^{n-1}\right)-E I\left(\boldsymbol{x}_{k}\right)^{\prime \prime \prime \prime}
$$

This is a semi-discretized Robin condition (14) with the choice of $\alpha_{f}$ given by (26). Notice that Eq. (27) is compatible with Eq. (25), the only difference being the presence of the flexural stiffness term that was neglected in the simplified model.

\section{The fully discrete problem}

We present the fully discrete problem for the case of the fluid problem (3),(4) with Robin boundary condition (24), and recall that a similar approach can be taken for the DN algorithm, since it is a particular case of the RN algorithm. We will state the problem in weak form by including only the boundary condition on $\Gamma(t)$, since those on $\partial \Omega_{f}(t) \backslash \Gamma(t)$ are understood and do not affect the presented methododology.

\subsection{The discrete fluid sub-problem}

For any given $t \in[0, T)$, we define the following spaces:

$$
\begin{aligned}
& V(t)=\left\{\boldsymbol{v}: \Omega_{f}(t) \rightarrow \mathbb{R}^{2}, \boldsymbol{v}=\hat{\boldsymbol{v}} \circ(\mathcal{A})^{-1}, \hat{\boldsymbol{v}} \in\left(H^{1}\left(\hat{\Omega}_{f}\right)\right)^{2}\right\}, \\
& Q(t)=\left\{q: \Omega_{f}(t) \rightarrow \mathbb{R}, q=\hat{q} \circ(\mathcal{A})^{-1}, \hat{q} \in L^{2}\left(\hat{\Omega}_{f}\right)\right\} .
\end{aligned}
$$

In the following we will use the notation $V^{n}:=V\left(t^{n}\right)$ and $Q^{n}:=Q\left(t^{n}\right)$ to denote the finite element spaces at the time instant $t^{n}$. 
We introduce the following linear forms:

$$
\begin{aligned}
m(\Omega ; \boldsymbol{u}, \boldsymbol{v}) & =\int_{\Omega}(\boldsymbol{u} \cdot \boldsymbol{v}) \mathrm{d} \Omega, \quad a(\Omega ; \boldsymbol{u}, \boldsymbol{v})=\int_{\Omega} \mu(\boldsymbol{\epsilon}(\boldsymbol{u}): \boldsymbol{\epsilon}(\boldsymbol{v})) \mathrm{d} \Omega, \\
c(\Omega ; \mathbf{w} ; \boldsymbol{u}, \boldsymbol{v}) & =\int_{\Omega}((\mathbf{w} \cdot \nabla) \boldsymbol{u} \cdot \boldsymbol{v}) \mathrm{d} \Omega, \quad b(\Omega ; p, \boldsymbol{v})=-\int_{\Omega} p \nabla \cdot \boldsymbol{v} \mathrm{d} \Omega .
\end{aligned}
$$

The variational formulation of the fluid problem $(3),(4)$ with boundary condition (14) reads: given $t \in(0, T]$, find $(\boldsymbol{u}, p) \in V(t) \times Q(t)$ such that $\forall(\boldsymbol{v}, q) \in V(t) \times Q(t)$ the following holds:

$$
\begin{aligned}
\rho_{f} m\left(\Omega_{f}(t) ;\left.\frac{\partial \boldsymbol{u}}{\partial t}\right|_{\hat{\mathbf{x}}}, \boldsymbol{v}\right)+\rho_{f} c\left(\Omega_{f}(t) ; \boldsymbol{u}-\mathbf{w} ; \boldsymbol{u}, \boldsymbol{v}\right)+a\left(\Omega_{f}(t) ; \boldsymbol{u}, \boldsymbol{v}\right) & +b\left(\Omega_{f}(t) ; p, \boldsymbol{v}\right) \\
+m\left(\Gamma(t) ; \alpha_{f} \boldsymbol{u}, \boldsymbol{v}\right) & =m\left(\Gamma(t) ; \alpha_{f} \dot{\boldsymbol{x}}-\boldsymbol{f}, \boldsymbol{v}\right), \\
b\left(\Omega_{f}(t) ; q, \boldsymbol{u}\right) & =0 .
\end{aligned}
$$

Time and space discretization. For simplicity, the implicit Euler scheme is used to discretize the above weak formulation in time. The convective term is linearized by a first order extrapolation formula. Notice that higher order discretization schemes and extrapolation formulas are also possible. At time $t^{n+1}$, and at the $(k+1)$-st RN sub-iteration, the time discrete linearized fluid sub-problem reads as follows: Find $\left(\boldsymbol{u}_{k+1}, p_{k+1}\right) \in V^{n} \times Q^{n}$ such that

$$
\begin{aligned}
\rho_{f} m\left(\Omega_{f}^{n} ; \frac{\boldsymbol{u}_{k+1}-\boldsymbol{u}^{n}}{\Delta t}, \boldsymbol{v}\right)+\rho_{f} c\left(\Omega_{f}^{n} ; \boldsymbol{u}_{k}-\mathbf{w}^{n} ; \boldsymbol{u}_{k+1}, \boldsymbol{v}\right)+a\left(\Omega_{f}^{n} ; \boldsymbol{u}_{k+1}, \boldsymbol{v}\right)+b\left(\Omega_{f}^{n} ; p_{k+1}, \boldsymbol{v}\right) \\
+m\left(\Gamma^{n} ; \alpha_{f} \boldsymbol{u}_{k+1}, \boldsymbol{v}\right)=m\left(\Gamma^{n} ; \alpha_{f} \dot{\boldsymbol{x}}_{k}-\boldsymbol{f}_{k}, \boldsymbol{v}\right), \\
b\left(\Omega_{f}^{n} ; q, \boldsymbol{u}_{k+1}\right)=0,
\end{aligned}
$$

for all $(\boldsymbol{v}, q) \in V^{n} \times Q^{n}$.

For the space discretization of problems (28)-(29), we choose the inf-sup stable Taylor-Hood finite element pair $\mathbb{P}_{2}-\mathbb{P}_{1}$. However, while the velocity field is continuous at $\Gamma^{n}$, the pressure space should be able to capture discontinuities across $\Gamma^{n}$, which are needed also for the correct evaluation of the hydrodynamic force (11). In order to deal with pressure discontinuities that occur at $\Gamma_{k}$, we use "node doubling" at the interface and apply a Subspace Projection Method to enforce the continuity of the velocity; see also [14, 13, 67] for a description of these techniques.

The linear system resulting from linearization and discretization is solved with the direct solver PARDISO $[56,74,75]$.

\subsection{The discrete structure problem}

For the time discretization of problem (5) or (7), we will consider a generalized Crank-Nicolson scheme [46]. At time $t^{n+1}$, Dirichlet-Neumann iteration $k+1$, the time discrete structure problem (7) is as follows: Find $\boldsymbol{x}_{k+1} \in K$ such that:

$$
\begin{aligned}
& \int_{0}^{L} \rho_{s} \frac{\boldsymbol{x}_{k+1}-2 \boldsymbol{x}^{n}+\boldsymbol{x}^{n-1}}{\Delta t^{2}} \cdot \boldsymbol{y} d s+E I \int_{0}^{L}\left(\alpha \boldsymbol{x}_{k+1}+(1-2 \alpha) \boldsymbol{x}^{n}+\alpha \boldsymbol{x}^{n-1}\right)^{\prime \prime} \cdot \boldsymbol{y}^{\prime \prime} d s \\
& =\int_{0}^{L}\left(\alpha \boldsymbol{f}_{k+1}+(1-2 \alpha) \boldsymbol{f}^{n}+\alpha \boldsymbol{f}^{n-1}\right) \cdot \boldsymbol{y} d s, \quad \forall \boldsymbol{y} \in d K\left(\boldsymbol{x}_{k+1}\right),
\end{aligned}
$$

where $K$ and $d K$ are defined in (8) and (9), respectively. Time discretization of problem (5) is similar. This scheme is known to be second order accurate for linear problems. For the numerical results in Sec. 6 , we will set $\alpha=1 / 4$ since in linear cases this choice leads to an unconditionally stable scheme, which possesses a very small numerical dissipation compared to other schemes, e.g., the Houbolt method [17, 11].

For the space discretization of problem (30), we use a third order Hermite finite element method [17]. To treat the inextensibility condition $\left|\boldsymbol{y}^{\prime}\right|=1$ in problem (IB), we use an augmented Lagrangian Method $[17,36,43,46,29]$. We refer to [11] and the references therein, for details. 


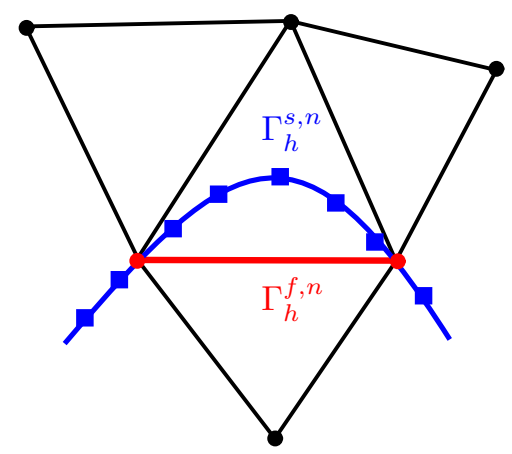

Figure 3: Fluid triangulation (black) aligned with the structure mesh $\Gamma_{h}^{s, n}$ (blue). The fluid nodes are marked with dots, while the structure nodes are marked with squares. $\Gamma_{h}^{f, n}$ (red) is the approximation of the interface given by an edge of the fluid mesh.

\subsection{Enforcement of the coupling conditions}

To describe the enforcement of the coupling conditions reported in Sec. 2.3 we first recall that at every time step the fluid mesh is aligned with the structure position. However, in general the fluid and structure meshes do not coincide, since they are made up of different elements: cubic Hermite elements on the structure side, and quadratic isoparametric edges on the fluid side. Due to the alignment, the fluid nodes that approximate the interface are always located on the structure mesh, as shown in Fig. 3. In Fig. 3, we denote by $\Gamma_{h}^{f, n}$ the approximation of the location of $\Gamma^{n}$ given by the fluid mesh, and by $\Gamma_{h}^{s, n}$ the approximation of $\Gamma^{n}$ by the structure mesh.

Enforcement of the kinematic coupling condition, i.e., the Dirichlet condition (20). Denote by $U_{\Gamma, k}$ and $\dot{X}_{k}$ the arrays of the nodal values of the corresponding fluid and structure velocities at the interface. Let $B_{f s}^{n}$ be the interpolation matrix of the structure mesh at the fluid interface nodes. To impose Dirichlet condition (20), we set

$$
U_{\Gamma, k+1}=B_{f s}^{n} \dot{X}_{k}
$$

Enforcement of the dynamic coupling condition. The fluid load onto the structure is given by the hydrodynamic force (11). The computation of the hydrodynamic force (11) is crucial for the numerical stability and accuracy of Domain Decomposition FSI solvers [33]. In the setting considered in this paper (an immersed beam), the quality of approximation of the pressure jump across the beam is of great importance, as demonstrated by the results in Sections 6.1 and 6.3.

The load exerted by the fluid onto the structure $\boldsymbol{f}_{\Gamma}$ can be computed as the variational residual $\mathcal{R}$ of the momentum conservation equation for the fluid, tested with test functions $\boldsymbol{v}$ that are different from zero on $\Gamma(t)$ :

$$
\begin{aligned}
\int_{\Gamma(t)} \boldsymbol{f}_{\Gamma} \cdot \boldsymbol{v} \mathrm{d} \Gamma & =-\int_{\Gamma(t)} \boldsymbol{\sigma}^{1} \boldsymbol{n}^{1} \cdot \boldsymbol{v} \mathrm{d} \Gamma-\int_{\Gamma(t)} \boldsymbol{\sigma}^{2} \boldsymbol{n}^{2} \cdot \boldsymbol{v} \mathrm{d} \Gamma \\
& =-\rho_{f} m\left(\Omega_{f}(t) ;\left.\frac{\partial \boldsymbol{u}}{\partial t}\right|_{\hat{\mathbf{x}}}, \boldsymbol{v}\right)-\rho_{f} c\left(\Omega_{f}(t) ; \boldsymbol{u}-\mathbf{w} ; \boldsymbol{u}, \boldsymbol{v}\right)-a\left(\Omega_{f}(t) ; \boldsymbol{u}, \boldsymbol{v}\right)-b\left(\Omega_{f}(t) ; p, \boldsymbol{v}\right) \\
& =\mathcal{R}\left(\Omega_{f}(t) ; \boldsymbol{u}, p, \boldsymbol{v}\right) .
\end{aligned}
$$

Let $\boldsymbol{f}_{\Gamma, k+1}^{f}$ denote the discrete hydrodynamic force at $\Gamma_{h}^{f, n}$ and sub-iteration $k+1$. After time and space discretization of (32), $\boldsymbol{f}_{\Gamma, k+1}^{f}$ is calculated from:

$$
\int_{\Gamma_{h}^{f, n}} \boldsymbol{f}_{\Gamma, k+1}^{f} \cdot \boldsymbol{v}_{h} \mathrm{~d} \Gamma=\mathcal{R}\left(\Omega_{f}^{n} ; \boldsymbol{u}_{h, k+1}, p_{h, k+1}, \boldsymbol{v}_{h}\right),
$$


where $\boldsymbol{u}_{h, k+1}$ and $p_{h, k+1}$ are the discrete velocity and pressure at the DN or RN sub-iteration $k+1$, obtained from solving system (28)-(29). By using matrix notation, eq. (33) can be written as follows:

$$
M_{\Gamma}^{n, f} F_{\Gamma, k+1}^{f}=R_{k+1},
$$

where $F_{\Gamma, k+1}^{f}$ is the array of nodal values of $\boldsymbol{f}_{\Gamma, k+1}^{f}, M_{\Gamma}^{n, f}$ is the mass matrix at $\Gamma_{h}^{f, n}$, and $R_{k+1}$ corresponds to the known values of the combined residuals appearing on the right-hand side of equation (33). This defines the hydrodynamic force, calculated at the fluid mesh nodes along the beam.

To enforce the dynamic coupling condition (13), this hydrodynamic force needs to be set equal to the structural load $f$ exerted onto the structure. For this purpose, we need to assign the values of the hydrodynamic force to the structure mesh nodes $\Gamma_{h}^{s, n}$ which do not necessarily lie on the structure discretization defined by the fluid mesh, see Fig. 3. To do that, we first project the structure mesh nodes of $\Gamma_{h}^{s, n}$ onto the fluid mesh interface $\Gamma_{h}^{f, n}$. At the fluid mesh, the fluid loading onto the structure $\boldsymbol{f}_{\Gamma, k+1}^{f}$ is defined by the process described above. We take those values of $\boldsymbol{f}_{\Gamma, k+1}^{f}$ and interpolate them first along the edges of the fluid mesh at the projected structural nodes, and then we assign those values back to the original structure nodes. More precisely, whenever the structural load $\boldsymbol{f}_{k+1}(\mathbf{x})$ needs to be evaluated for some $\mathbf{x} \in \Gamma_{h}^{s, n}$ (for instance at the quadrature nodes needed to evaluate the right hand side of eq. (30)), we first define the projected structure node

$$
\tilde{\mathbf{x}}:=\underset{\mathbf{y} \in \Gamma_{h}^{f, n}}{\arg \min }\|\mathbf{x}-\mathbf{y}\|
$$

and then let $\boldsymbol{f}_{k+1}(\mathbf{x})=\boldsymbol{f}_{\Gamma, k+1}^{f}(\tilde{\mathbf{x}})$. We use the following notation to summarize this procedure:

$$
F_{\Gamma, k+1}^{s}=B_{s f}^{n} F_{\Gamma, k+1}^{f},
$$

where we used $B_{s f}^{n}$ to denote the extrapolation of the values of the hydrodynamic quantities at the fluid nodes onto the the structure nodes. This defines the hydrodynamic force at the structure mesh nodes, and enforces the dynamic coupling condition (13).

It is important to notice that in this numerical implementation of the dynamic coupling condition, the power exchanged between the fluid and structure is not perfectly balanced, i.e., at the discrete level, the energy imparted by the fluid onto the structure is not perfectly converted into the total energy of the structure, and vice versa. This is due to the non-matching fluid and structure meshes. In the case of the DN algorithm, this mismatch can be precisely quantified as follows.

At the time $t^{n+1}$, after the convergence of the DN sub-iterations, the discrete power exchanged at the interface from the fluid side is

$$
P^{f, n+1}=\int_{\Gamma_{h}^{f, n+1}} \boldsymbol{f}_{\Gamma}^{f, n+1} \cdot \boldsymbol{u}_{h}^{n+1} \mathrm{~d} \Gamma=\left(U_{\Gamma}^{n+1}\right)^{T} M_{\Gamma}^{f, n+1} F_{\Gamma}^{f, n+1}=\left(\dot{X}^{n+1}\right)^{T}\left(B_{f s}^{n+1}\right)^{T} M_{\Gamma}^{f, n+1} F_{\Gamma}^{f, n+1},
$$

where for the last equation we used (31). Similarly, the discrete power exchanged at the interface from the structure side is

$$
P^{s, n+1}=\int_{\Gamma_{h}^{s, n+1}} \boldsymbol{f}_{\Gamma}^{s, n+1} \cdot \dot{\boldsymbol{x}}_{h}^{n+1} \mathrm{~d} \Gamma=\left(\dot{X}^{n+1}\right)^{T} M_{\Gamma}^{s, n+1} F_{\Gamma}^{s, n+1}=\left(\dot{X}^{n+1}\right)^{T} M_{\Gamma}^{s, n+1} B_{s f}^{n+1} F_{\Gamma}^{f, n+1},
$$

where for the last equation we used (35). Thus, the power exchanged at the interface is balanced if

$$
\left(B_{f s}^{n+1}\right)^{T} M_{\Gamma}^{f, n+1}=M_{\Gamma}^{s, n+1} B_{s f}^{n+1} .
$$

Since $\Gamma_{h}^{f, n+1}$ and $\Gamma_{h}^{s, n+1}$ are aligned but do not coincide $\left(\Gamma_{h}^{s, n+1}\right.$ is a piecewise cubic globally $C^{1}$ function, and $\Gamma_{h}^{f, n+1}$ is a piecewise quadratic interpolation) and the fluid and structure discretizations are based on different elements, the balance equation is not necessarily fulfilled exactly. However, in Sec. 6.5.2 we 
will show that the difference between $P^{f, n+1}$ and $P^{s, n+1}$ is very small $(0.01 \%$ of the power value) in our computations.

\section{Enforcement of Robin boundary condition (27).}

We first evaluate the right hand side of the Robin boundary condition (27) on the structure mesh. Denote by $S_{k}$ the discretization of the right hand side at sub-iteration $k$. Then $S_{k}$ is given by:

$$
\int_{0}^{L} S_{k} \cdot \boldsymbol{y} d s=\int_{0}^{L} \rho_{s} \frac{\boldsymbol{x}^{n}-\boldsymbol{x}^{n-1}}{\Delta t^{2}} \cdot \boldsymbol{y} d s-E I \int_{0}^{L}\left(\alpha \boldsymbol{x}_{k}+(1-2 \alpha) \boldsymbol{x}^{n}+\alpha \boldsymbol{x}^{n-1}\right)^{\prime \prime} \cdot \boldsymbol{y}^{\prime \prime} d s .
$$

Boundary condition (27) can be treated similar to (31) by interpolating $S_{k}$ at the fluid interface nodes. Thus, the Robin boundary condition (27) in matrix form reads:

$$
\frac{\rho_{s}}{\Delta t} M_{\Gamma}^{f, n} U_{\Gamma, k+1}-M_{\Gamma}^{f, n} F_{\Gamma, k+1}^{f}=M_{\Gamma}^{f, n} B_{f s}^{n} S_{k}
$$

\section{Numerical results}

A series of numerical tests is presented that showcase the main features and performance of the Extended ALE Method. In all the tests, the fluid density of $\rho_{f}=1 \mathrm{~g} / \mathrm{cm}^{3}$ is considered, the structure mesh always consists of 45 nodes (with cubic Hermite elements), and the stopping tolerance in (21) for the partitioned schemes (either DN or RN) is always set to $\varepsilon=10^{-8}$. We use the SI unit system, and present all the quantities in the centimeter-gram-second (CGS) units. If the units of a certain quantity are omitted for the sake of simplicity, it is implied that they are in the CGS system.

\subsection{A stationary periodic beam}

The goal of this first test is to show the importance of accurately capturing the pressure discontinuity across the interface, and to validate our implementation of the Extended ALE Method and RN algorithm. We choose a simple test case with a stationary solution, which can be calculated explicitly.

We consider the periodic beam model $(\mathbf{P B})$ with $E I=1 \mathrm{~g} /\left(\mathrm{cm} \mathrm{s}^{2}\right), \rho_{s}=10 \mathrm{~g} / \mathrm{cm}$, and we take the beam to be a circle of radius 1 centered at the origin:

$$
\boldsymbol{x}(s)=\left[\begin{array}{ll}
\cos (s) & \sin (s)
\end{array}\right]^{T}, \quad s \in[0,2 \pi],
$$

immersed in a viscous fluid with viscosity $\mu=1 \mathrm{~g} / \mathrm{cm} \mathrm{s}$, occupying a rectangular fluid domain $\Omega_{f}=$ $(-2,2) \times(-2,2)$. The fluid is at rest, i.e. $\boldsymbol{u}=\mathbf{0}$.

The coupled FSI problem (3) through (6), plus (12) and (13), has a steady-state solution, which is given by Eq. (40) for the structure position and

$$
\boldsymbol{u}=\mathbf{0} \quad \text { in } \quad \Omega_{f}, \quad p(\boldsymbol{x})=\left\{\begin{array}{lll}
E I & \text { if } & \boldsymbol{x} \in \Omega_{f}^{1} \\
0 & \text { if } & \boldsymbol{x} \in \Omega_{f}^{2}
\end{array}\right.
$$

where $\Omega_{f}^{1}\left(\Omega_{f}^{2}\right)$ is the fluid domain inside (outside) the beam.

We fix the time step size to be $\Delta t=10^{-2} \mathrm{~s}$ and consider four different fluid meshes of mesh size

$$
h_{j}=0.25 \cdot 2^{-j}, \quad j=1, \ldots, 4 .
$$

We solve the time-dependent problem (3), (4), (5), (6), (12), (13), with the initial data (40) and $\boldsymbol{u}=\mathbf{0}$. We consider two different pressure approximations: a discontinuous and a continuous one. Table 1 reports the errors for the velocity in the $H^{1}$ semi-norm, and for the pressure in the $L^{2}$ norm, after 100 time steps. 


\begin{tabular}{|c||c|c||c|c|}
\hline \multicolumn{1}{|c||}{} & \multicolumn{2}{c||}{ continuous $p$} & \multicolumn{2}{c|}{ discontinuous $p$} \\
\hline ref. level $j$ & $\left|\boldsymbol{u}_{h}-\boldsymbol{u}\right|_{1}$ & $\left\|p_{h}-p\right\|$ & $\left|\boldsymbol{u}_{h}-\boldsymbol{u}\right|_{1}$ & $\left\|p_{h}-p\right\|$ \\
\hline 1 & $0.54681 e+00$ & $0.25786 e+00$ & $0.63169 e-05$ & $0.43490 e-05$ \\
2 & $0.37705 e+00$ & $0.18234 e+00$ & $0.69300 e-06$ & $0.25414 e-06$ \\
3 & $0.27219 e+00$ & $0.13316 e+00$ & $0.91836 e-06$ & $0.20680 e-06$ \\
4 & $0.19135 e+00$ & $0.93794 e-01$ & $0.93755 e-06$ & $0.18234 e-06$ \\
\hline
\end{tabular}

Table 1: $L^{2}$ errors for the pressure and errors in the $H^{1}$ semi-norm for the velocity after 100 time steps obtained by using continuous and discontinuous pressure approximations on four meshes with different refinement levels.

In the continuous pressure case, we observe a low convergence rate of about $h^{1 / 2}$, which is to be expected when approximating discontinuous functions using continuous functions (see for instance [47]). The same holds for the velocity in the $H^{1}$ semi-norm, indicating the presence of spurious velocities which often lead to instabilities. When using a discontinuous approximation for the pressure, the approximation error is reduced significantly. In fact, already for refinement level 2 , we reach a value of the order of $10^{-6}$.

Figure 4 shows a warp of the pressure obtained with both continuous and discontinuous pressure approximations for refinement level 2: spurious oscillations appear in the case of a continuous pressure approximation.

This example shows that our methodology captures the correct steady-state solution, and it emphasizes the importance of accurate pressure approximation for this class of problems, motivating the use of a discontinuous pressure approximation, which we employ in the rest of this work.

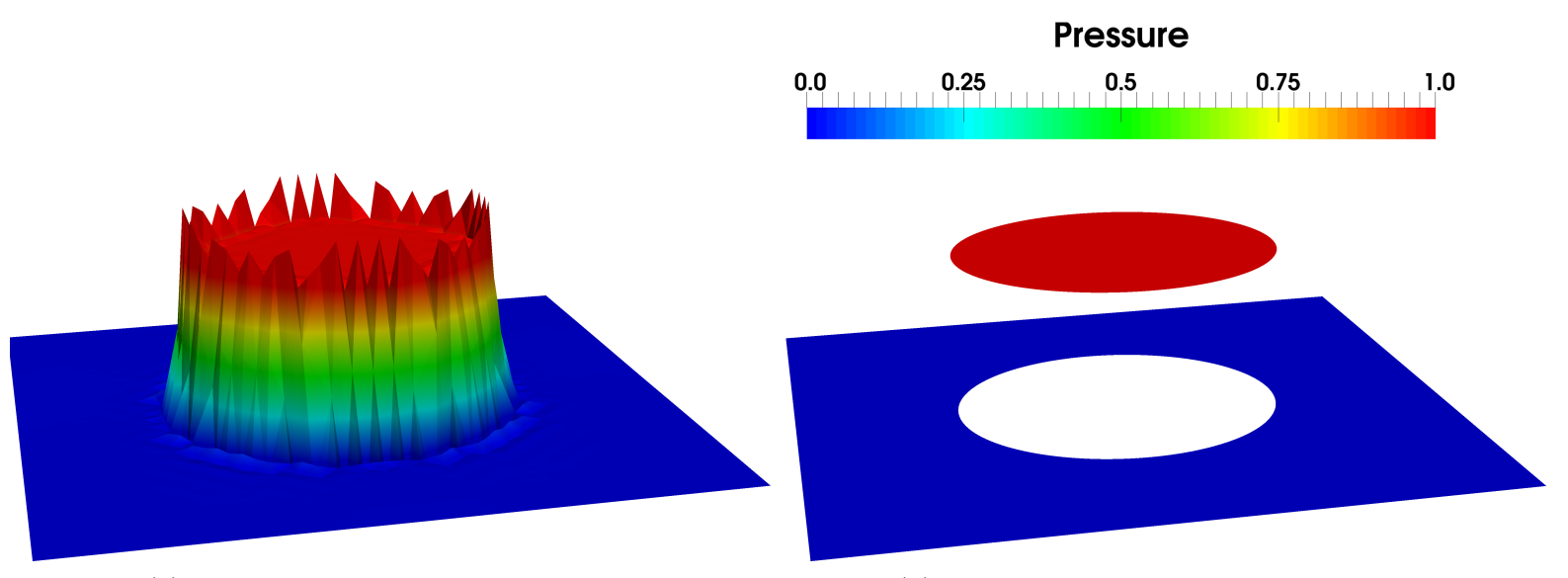

(a) Continuous pressure approximation

(b) Discontinuous pressure approximation

Figure 4: Warp of the pressure solution after 100 time steps for (a) continuous and (b) discontinuous pressure approximations on the mesh with refinement level 2.

\subsection{A rotating periodic beam}

The second test is quasi-stationary: we consider a rotation of the periodic structure in the previous subsection. The geometry is the same as in subsection 6.1. The structure parameters are set to $E I=1$ $\mathrm{g} /\left(\mathrm{cm} \mathrm{s}^{2}\right)$ and $\rho_{s}=1 \mathrm{~g} / \mathrm{cm}$. Observe that

$$
\boldsymbol{u}(t, \boldsymbol{x})=\boldsymbol{u}(x, y)=\left(\begin{array}{c}
-y \\
x
\end{array}\right), \quad p(x, y)=0.5\left(x^{2}+y^{2}\right) \quad \text { in } \quad \Omega_{f}
$$

defines a quasi-stationary solution to the coupled FSI problem (3) through (6), plus (12) and (13). A short computation reveals that the kinematic coupling condition

$$
\boldsymbol{u}(t, \boldsymbol{x}(t, s))=\dot{\boldsymbol{x}}(t, s) \quad \text { on } \Gamma(t)
$$


implies

$$
\begin{aligned}
\rho_{s} \ddot{\boldsymbol{x}}(t, s) & =\rho_{s} \frac{\mathrm{d}}{\mathrm{dt}} \boldsymbol{u}(t, \boldsymbol{x}(t, s))=\rho_{s}\left(\partial_{t} \boldsymbol{u}(t, \boldsymbol{x}(t, s))+\nabla \boldsymbol{u}(t, \boldsymbol{x}(t, s)) \dot{\boldsymbol{x}}(t, s)\right) \\
& =\rho_{s} \nabla \boldsymbol{u}(t, \boldsymbol{x}(t, s)) \boldsymbol{u}(t, \boldsymbol{x}(t, s))=-\rho_{s} \boldsymbol{x}(t, s)
\end{aligned}
$$

and thus

$$
\rho_{s} \ddot{\boldsymbol{x}}+E I \boldsymbol{x}^{\prime \prime \prime \prime}=\mathbf{0}=\boldsymbol{f}_{\Gamma},
$$

for our choice of $\rho_{s}$ and $E I$.

Observe that even though the fluid solution (42) is stationary with respect to time, the kinematic boundary condition leads to a time-dependent solution of the structure problem, i.e. a counter clockwise rotation of the structure.

We fix the time step size to be $\Delta t=10^{-4} \mathrm{~s}$ to keep time discretization errors as small as possible and consider four different fluid meshes of mesh size

$$
h_{j}=0.25 \cdot 2^{-j}, \quad j=1, \ldots, 4 .
$$

We solve the time-dependent problem (3), (4), (5), (6), (12), (13), with the initial data for $\boldsymbol{u}$ and $\boldsymbol{x}$ corresponding to the above solution. The errors of the pressure (in the $L^{2}$ norm) and the velocity (in the $H^{1}$ semi-norm and $L^{2}$ norm) obtained after 100 time steps are shown in Table 2.

\begin{tabular}{|c||c|c||c|c||c|c|}
\hline ref. level $j$ & $\left|\boldsymbol{u}_{h}-\boldsymbol{u}\right|_{1}$ & EOC & $\left\|\boldsymbol{u}_{h}-\boldsymbol{u}\right\|$ & EOC & $\left\|p_{h}-p\right\|$ & EOC \\
\hline 1 & $0.41993 e-02$ & - & $0.26108 e-03$ & - & $0.96069 e-01$ & - \\
2 & $0.72518 e-03$ & 2.53 & $0.23023 e-04$ & 3.50 & $0.27127 e-01$ & 1.82 \\
3 & $0.11887 e-03$ & 2.61 & $0.22112 e-05$ & 3.38 & $0.65248 e-02$ & 2.06 \\
4 & $0.24062 e-04$ & 2.30 & $0.67627 e-06$ & 1.71 & $0.15898 e-02$ & 2.04 \\
\hline
\end{tabular}

Table 2: Errors in the $H^{1}$ semi-norm and $L^{2}$ norm for the velocity and $L^{2}$ errors for the pressure after 100 time steps obtained on three different mesh sizes using a fixed time step size of $\Delta t=10^{-4} \mathrm{~s}$.

While we observe the theoretically expected convergence rate of 2 for the pressure in the $L^{2}$ norm, the velocity errors show a slightly super-convergent behavior. This is to be expected, since the linear velocity solution is only "almost" contained in the velocity trial space which is made up of isoparametric deformed elements at the structure interface which do not necessarily contain piecewise linear functions.

\subsection{An oscillating periodic beam}

The goals of this example are: (1) to show that for small structural displacements, the solution obtained using our Extended ALE Method "coincides" with the solution obtained using the standard ALE approach, and (2) to check the convergence behavior of our approach in time and space.

We consider the same fluid domain as in the previous test, but we decrease the fluid viscosity to $\mu=0.2$ $\mathrm{g} / \mathrm{cm} \mathrm{s}$. The fluid is initially at rest. The initial shape of the beam is elliptic:

$$
\boldsymbol{x}(s)=[a \cos (s), \sin (s) / a]^{T}, \quad s \in[0,2 \pi],
$$

where $a>0$ is a given parameter. The no-slip condition is imposed at the rectangular (rigid) boundary of the fluid domain $\Omega_{f}$.

Due to elastic forces, we expect the beam to evolve towards the steady state solution of a circle, with an area equivalent to that of the initial ellipse (46), i.e. a circle of radius 1 . We choose the parameter $a=1.5$ such that the structure displacement is not too large, and the standard ALE method can be applied to simulate the problem. 

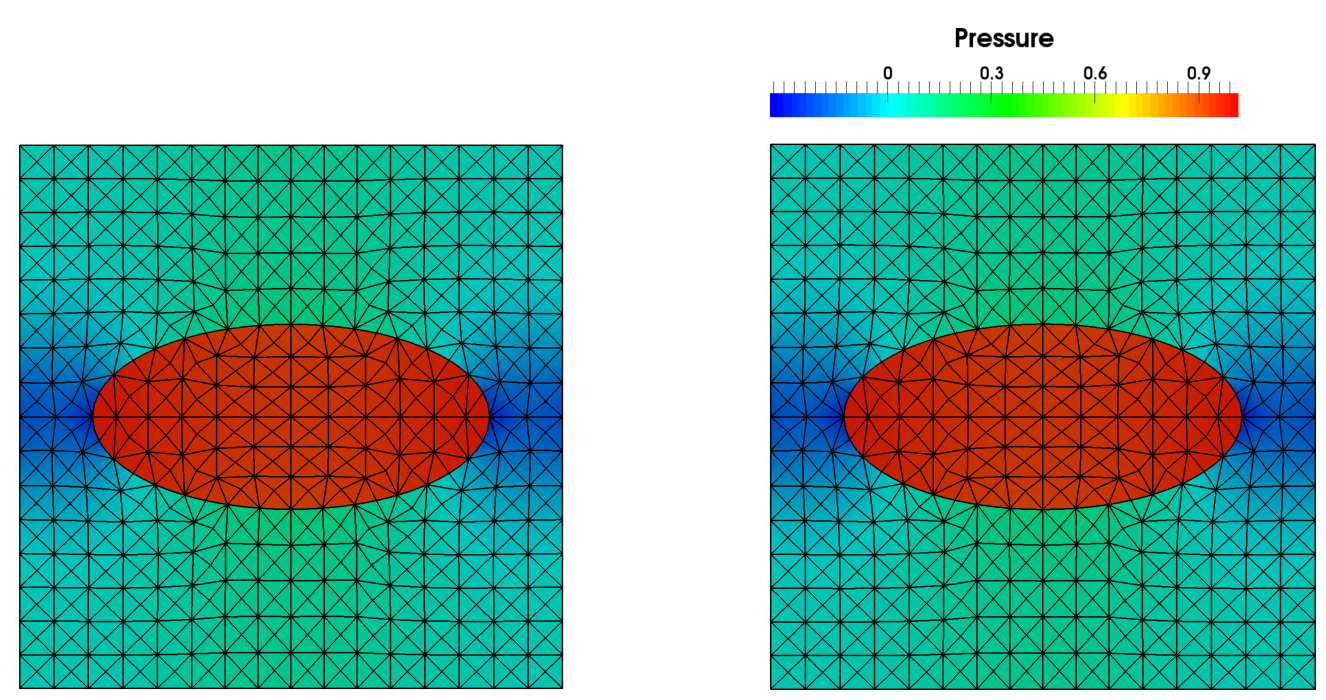

(a) $t=1 \mathrm{~s}$
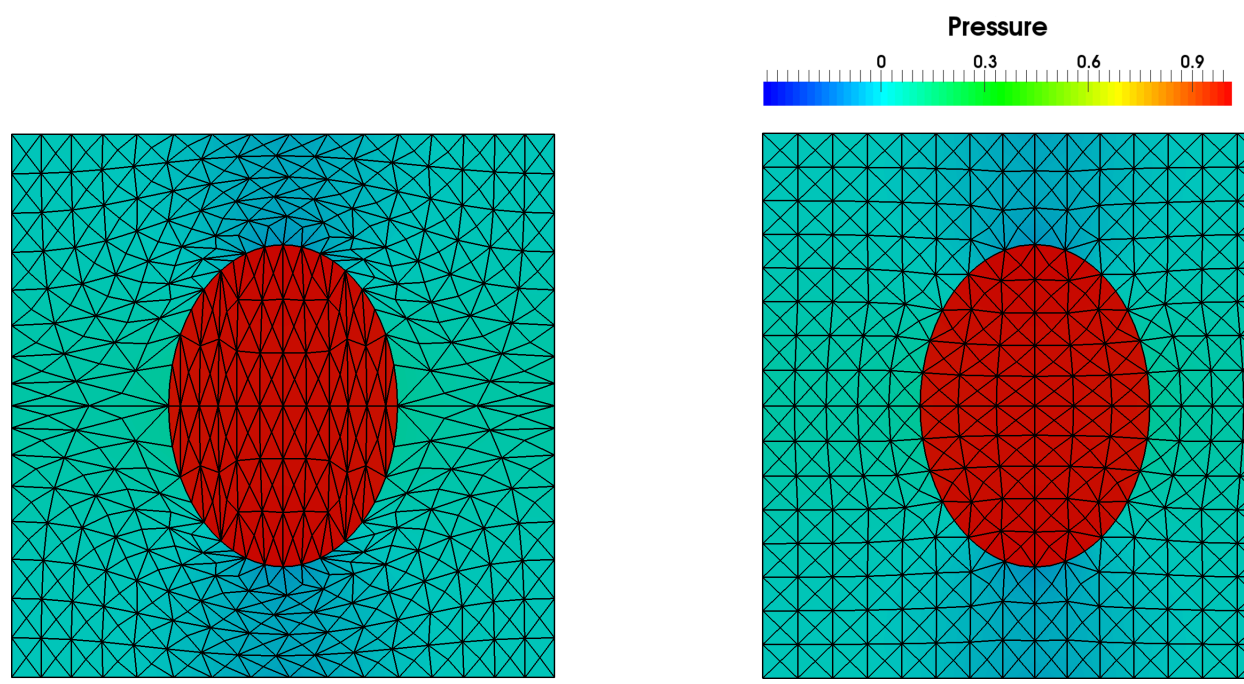

(b) $t=10 \mathrm{~s}$
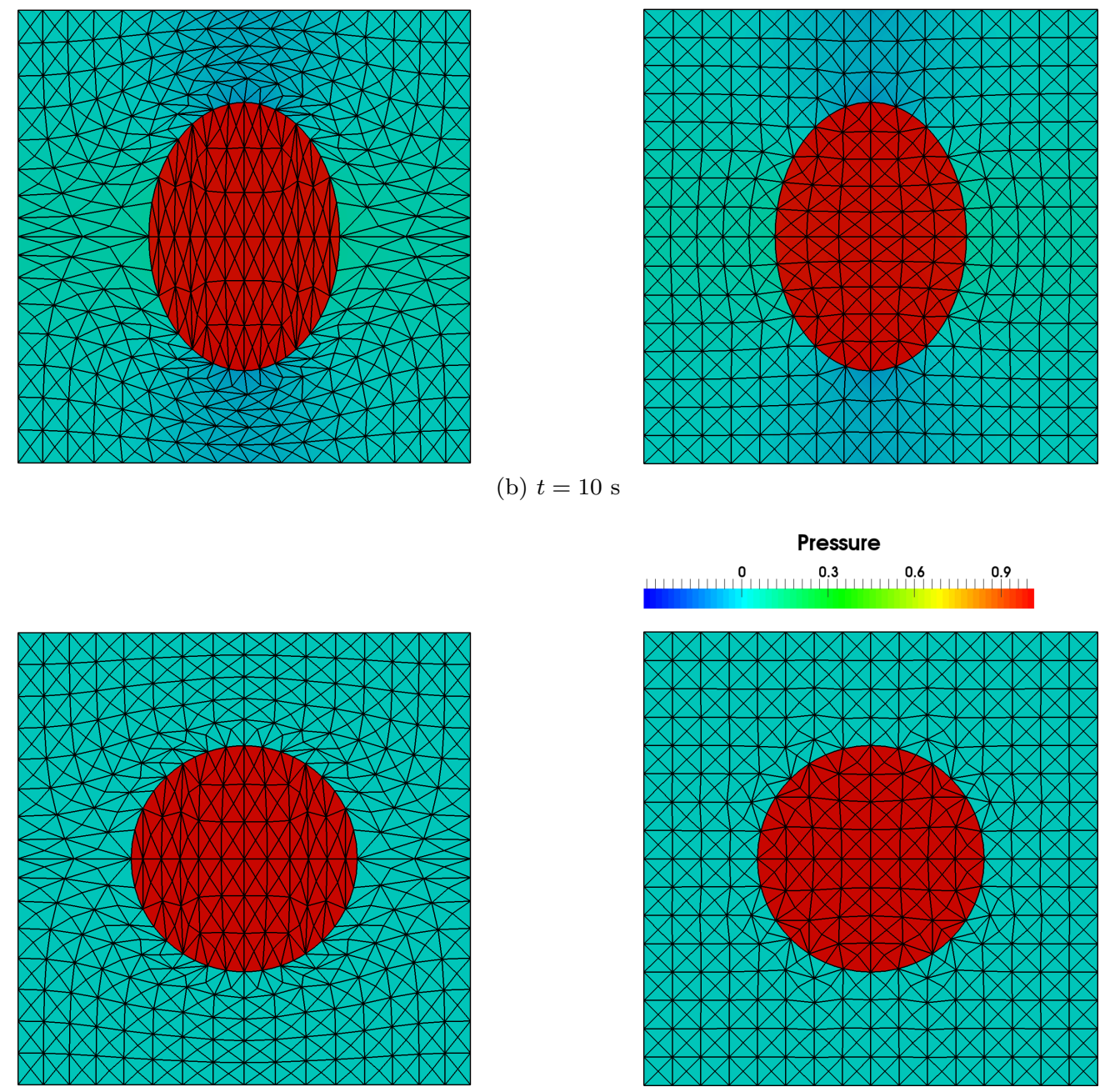

(c) $t \overline{\overline{19}} 100 \mathrm{~s}$

Figure 5: Pressure and (coarse) mesh deformation for the oscillating beam at time (a) $t=1 \mathrm{~s}$, (b) $t=10 \mathrm{~s}$, and (c) $t=100 \mathrm{~s}$ for both the standard ALE (left) and extended ALE methods (right). 
Fig. 5 shows the computed pressure and mesh deformation at times $t=1 \mathrm{~s}, 10 \mathrm{~s}$, and $100 \mathrm{~s}$ for both standard ALE and extended ALE methods. We see in Fig. 5(a) and (b) that the beam, initially elliptic, oscillates. Then, at time $t=100 \mathrm{~s}$ it reaches a circular shape, shown in Fig. 5(c). These figures also show the difference in the mesh deformation given by the standard ALE method and our Extended ALE approach.

Next, we track the maximum $x$-coordinate of the beam position over time for:

- two mesh sizes $h=0.25 \cdot 2^{-j}$, with $j=0$ (coarse mesh) and $j=1$ (fine mesh), and

- four time step sizes $\Delta t=0.1 \cdot 2^{-j}, j=0, \ldots, 3$.

The maximum $x$-coordinate is expected to evolve towards 1 through damped oscillations, given that the stationary solution for the beam is the circle of radius 1 centered at the origin. 


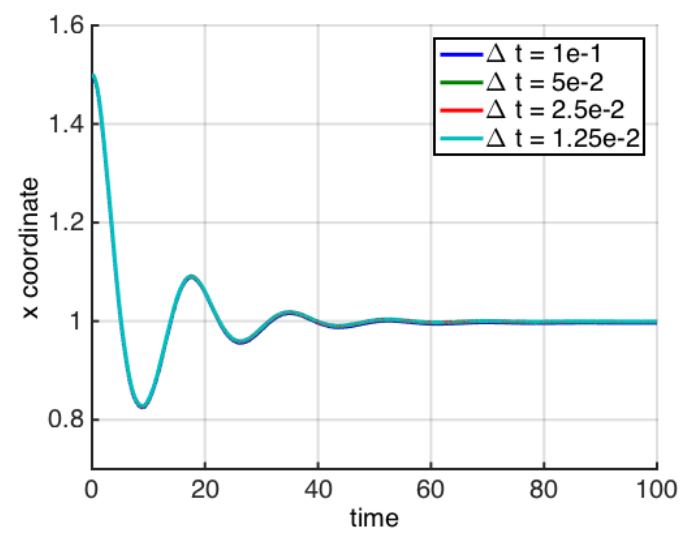

(a) standard ALE, $j=0$ (coarse mesh)

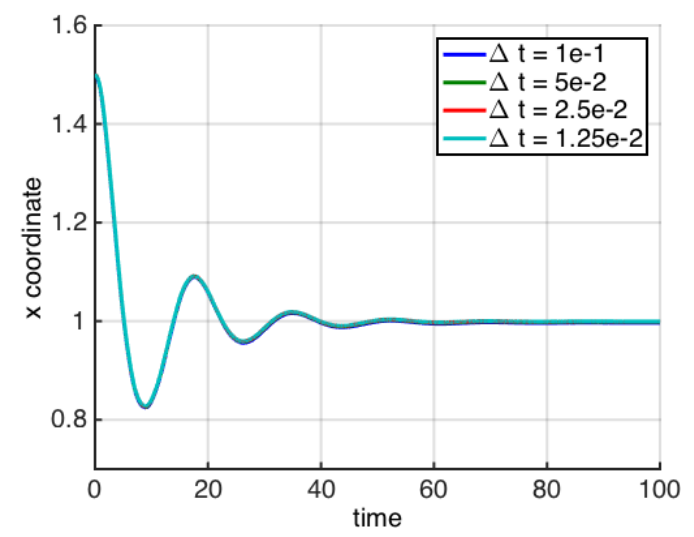

(c) standard ALE, $j=1$ (fine mesh)

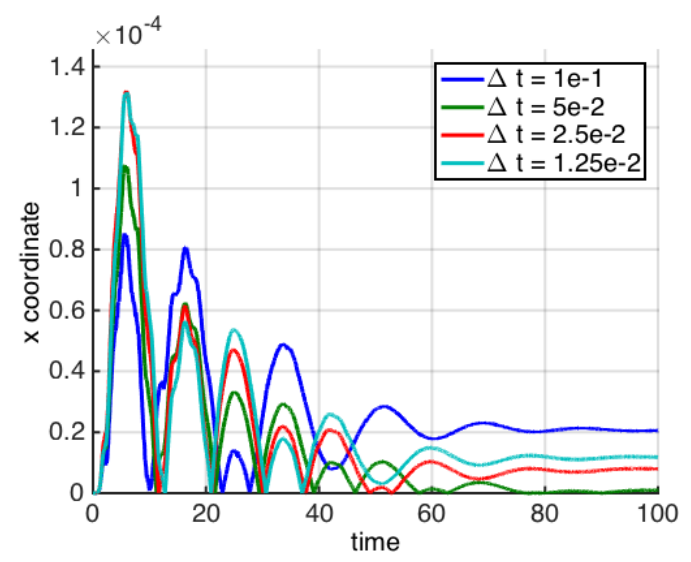

(e) difference between standard and Extended for $j=0$ (coarse mesh)

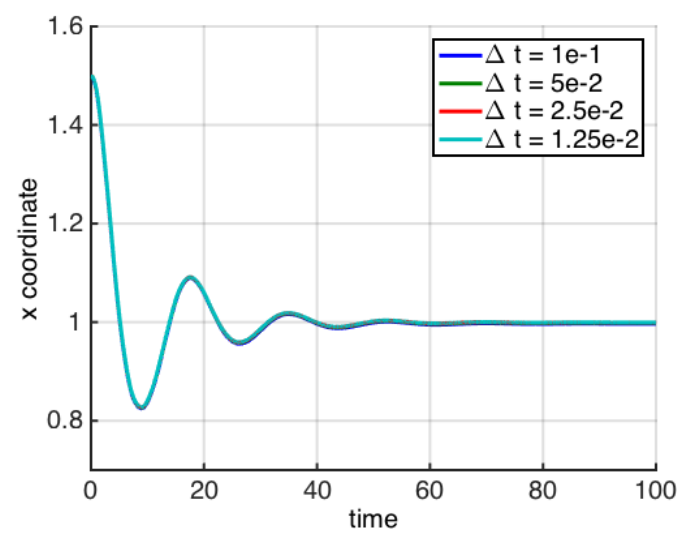

(b) Extended ALE, $j=0$ (coarse mesh)

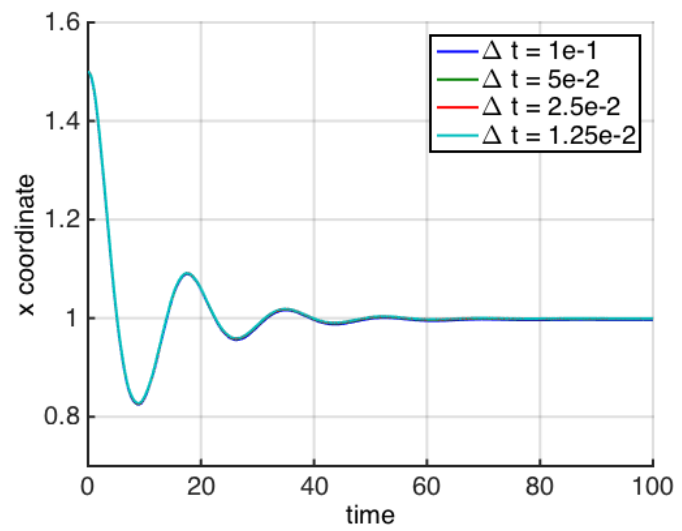

(d) Extended ALE, $j=1$ (fine mesh)

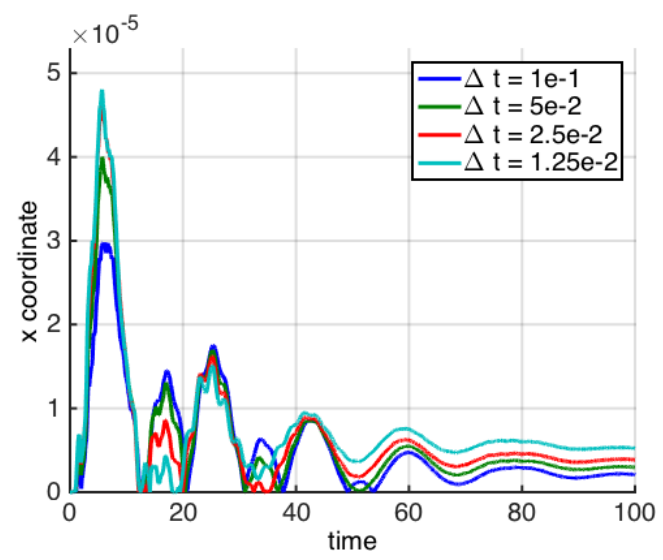

(f) difference between standard and Extended for $j=1$ (fine mesh)

Figure 6: Maximum $x$-coordinate of the beam position (in $\mathrm{cm}$ ) over time (in s) for (a) standard ALE and coarse mesh, (b) Extended ALE and coarse mesh, (c) standard ALE and fine mesh, and (d) Extended ALE and fine mesh. (e) Difference in absolute value between the curves in (a) and (b), and (f) difference in absolute value between the curves in (c) and (d).

Indeed, Fig. 6(a) and (b) display these damped oscillations computed with the coarse mesh for all the 
time steps under consideration by the standard and Extended ALE method, respectively. The corresponding figures for the fine mesh are Figs. 6(c) and (d). For both methods, both meshes, and all the time steps, the maximum $x$-coordinate evolves towards 1 , as expected. Moreover, we see that the curves obtained with the different time steps are almost superimposed over the whole time interval for both methods and both meshes, indicating that we have reached time step size independence for a rather large interval of time. Fig. 6(e) shows the difference in absolute value between the curves in Fig. 6(a) and (b), while the difference in absolute value between the curves in Fig. 6(c) and (d) is reported in Fig. 6(f). From Fig. 6(e) and (f) we see that there is a slight difference in amplitude between the oscillations computed by the standard and Extended ALE methods. Such a difference should get smaller as the mesh gets finer. In fact, the maximum difference in the maximum $x$-coordinate is around $1.3 \cdot 10^{-4}$ for the coarse mesh and around $5 \cdot 10^{-5}$ for the fine mesh, indicating that the beam maximum $x$-coordinates computed by the two methods get closer with mesh refinement.

\subsection{Periodic beam advected by a channel flow}

This example is designed to show that for FSI problems with large deformation, the standard ALE method breaks down due to excessive mesh distortion, while the Extended ALE Method keeps the mesh quality under control, and gives excellent results beyond the point of break down of standard ALE.

We consider the fluid domain to be a rectangular channel $\Omega_{f}=(-12,12) \times(-2,2)$ filled with a viscous fluid of viscosity $\mu=0.1 \mathrm{~g} / \mathrm{cm}$. At $t=0$, the fluid is at rest, and the beam of circular shape, with radius 1 , is immersed in the fluid, with its center located at $(-8,0)$. See Fig. $7(\mathrm{a})$. The following structure parameters are used in the simulation: $\rho_{s}=10 \mathrm{~g} / \mathrm{cm}$ and $E I=1 \mathrm{~g} /\left(\mathrm{cm} \mathrm{s}^{2}\right)$.

We study FSI between the periodic beam and the flow of a viscous, incompressible fluid, which is driven by the boundary conditions imposed on $\Gamma_{i n}$ and $\Gamma_{\text {out }}$ (see Fig. 1(a)). At $\Gamma_{i n}$, we prescribe a non-zero $x$ component of the fluid velocity, which is smoothly increased to $1 \mathrm{~cm} / \mathrm{s}$ over time interval [0,1], and is kept equal to $1 \mathrm{~cm} / \mathrm{s}$ until $t=20 \mathrm{~s}$, when it drops to $0 \mathrm{~cm} / \mathrm{s}$ :

$$
\left.\boldsymbol{u}(t, \boldsymbol{x})\right|_{\Gamma_{i n}}= \begin{cases}{\left[-2 t^{3}+3 t^{2}, 0\right]^{T}} & \text { if } 0 \leq t \leq 1 \\ {[1,0]^{T}} & \text { if } 1 \leq t \leq 20 \\ {[0,0]^{T}} & \text { if } t>20\end{cases}
$$

At $\Gamma_{\text {out }}$ we enforce a homogeneous Neumann boundary condition. The no-slip condition is prescribed at the top and bottom channel boundary.

Due to the background fluid flow, the periodic beam is transported through the channel while undergoing strong deformation as the fluid and structure interact via a two-way coupling. See Fig. 7(b) and (c). At $t \geq 20 \mathrm{~s}$ when the inlet fluid velocity returns to zero, the viscous forces lead to a deceleration of the beam, which stops and eventually returns to its original circular shape, as visible at around $t=100 \mathrm{~s}$. See Fig. $7(\mathrm{f})$.

Figure 7 shows the beam position and mesh deformation computed at different times by the standard ALE method (top panel in each subfloat) and the Extended ALE method (bottom panel in each subfloat). The standard ALE is able to follow the deformation and transport of the beam until about $t \approx 18.45 \mathrm{~s}$, when mesh distortion becomes too severe. For this reason, the position of the beam in the top panels in Figs. 7(d), (e), and (f), corresponding to times $t=25,50$, and $100 \mathrm{~s}$, is not updated. As long as the two simulations run, we observe very good agreement in the beam position and deformation computed by the standard and Extended ALE methods. See Fig. 7(a), (b), and (c). A more detailed comparison between the two methods is shown in Fig. 8. There, a comparison between the maximum $x$-coordinate of the beam position computed by the two methods is given in Fig. 8(a), showing excellent agreement. Fig. 8(b) shows a close-up view of Fig. 8(a) around the time when the standard ALE method breaks down. Notice that until $t \approx 18.45 \mathrm{~s}$ the curves given by the two methods are almost superimposed. With the extended ALE method we are able to carry out the simulation all the way until $t=100 \mathrm{~s}$ when the fluid velocity and beam motion are almost zero, and the shape of the beam has returned to almost circular.

Fig. 7 shows that the mesh obtained with the standard ALE method gets severely distorted, while the quality of the mesh computed by our Extended ALE method remains high throughout the entire time 
Standard ALE

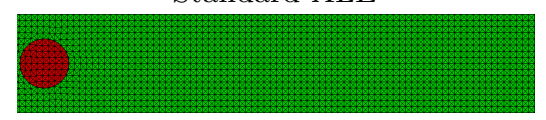

Extended ALE

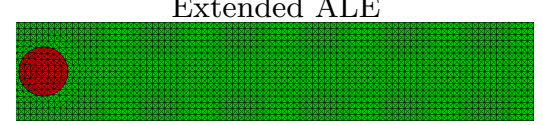

(a) $t=0 \mathrm{~s}$

Extended ALE

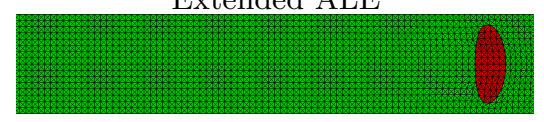

(d) $t=25 \mathrm{~s}$
Standard ALE

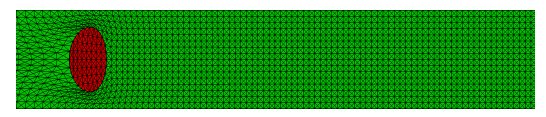

Extended ALE

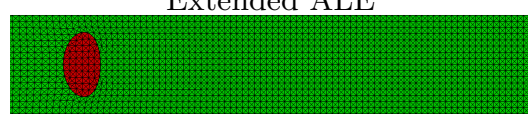

(b) $t=5 \mathrm{~s}$

Extended ALE

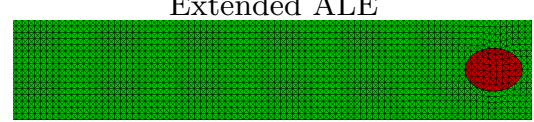

(e) $t=50 \mathrm{~s}$
Standard ALE

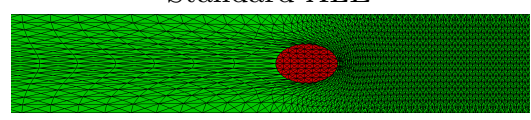

Extended ALE

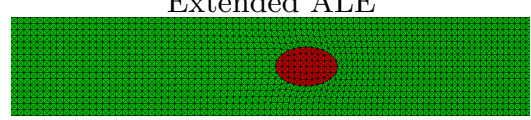

(c) $t=15 \mathrm{~s}$

Extended ALE

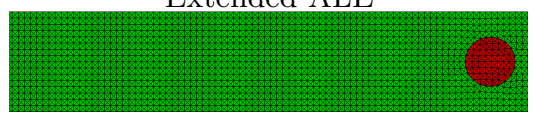

(f) $t=100 \mathrm{~s}$

Figure 7: Beam position and mesh deformation for the periodic beam transported through a channel at (a) $t=0 \mathrm{~s}$, (b) $t=5$ $\mathrm{s},(\mathrm{c}) t=15 \mathrm{~s}$, (d) $t=25 \mathrm{~s}$, (e) $t=50 \mathrm{~s}$, (f) $t=100 \mathrm{~s}$. When available, the solution computed by the standard ALE method is above the solution computed by the Extended ALE method.

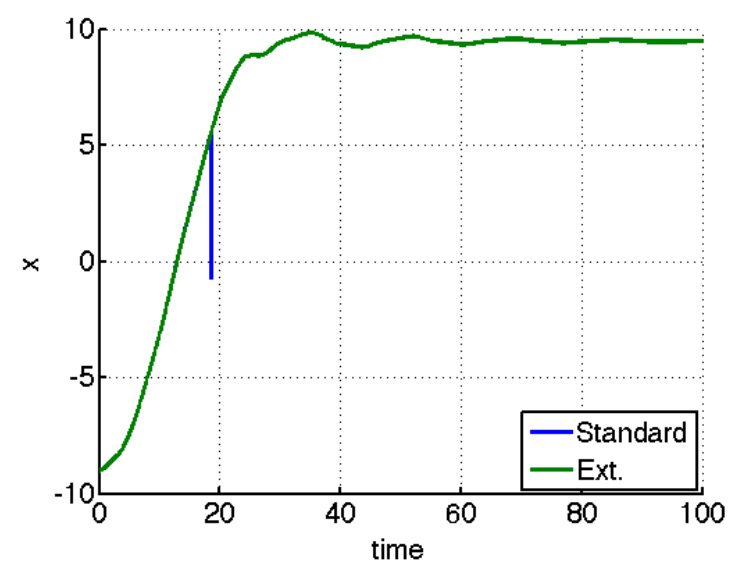

(a) Maximum $x$-coordinate of the beam

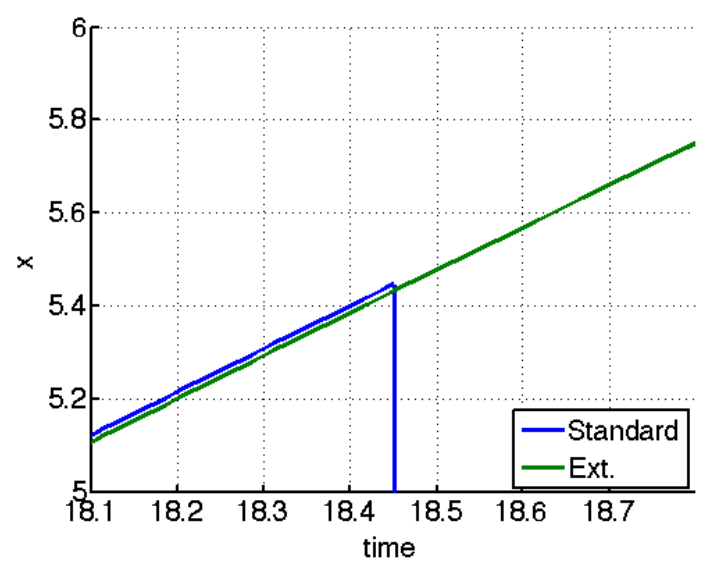

(b) Zoomed view

Figure 8: (a) Maximum $x$-coordinate of the beam position over time (in s) computed by the standard and Extended ALE methods and (b) close-up view around the time when the standard ALE method breaks down $(t=18.45 \mathrm{~s})$. 
interval. As a further proof of the different quality of the meshes given by the standard and extended ALE methods, we report in Fig. 9 the maximum angle of the mesh elements over time. We see that the maximum angle in the mesh given by the standard ALE method increases up to nearly 170 degrees, right before the simulation crashes. On the other hand, the maximum angle for the mesh given by the Extended ALE method never exceeds 132 degrees.

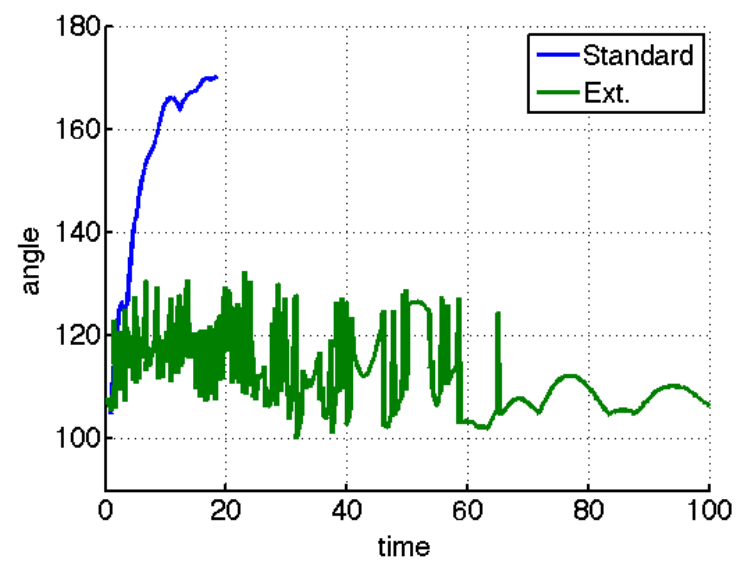

Figure 9: Maximum angle of the elements in the mesh given by the standard and Extended ALE methods versus time (in s).

\subsection{An immersed beam}

This test is aimed at assessing the performance of the DN algorithm, without and with Aitken's acceleration method, and the performance of RN algorithm. We consider the immersed beam model (IB), which is more challenging than the periodic beam model (PB) due to the inextensibility constraint [29]. For this test, we are going to use only the Extended ALE Method.

\subsubsection{Comparison between the $D N$ and $R N$ algorithms}

We consider a channel $\Omega_{f}=[-3,3] \times[-0.5,0.5]$ filled with a viscous, incompressible fluid of viscosity $\mu=0.01 \mathrm{~g} / \mathrm{m} \mathrm{s}$. A beam of length $0.5 \mathrm{~cm}$, with $\rho_{s}=5 \mathrm{~g} / \mathrm{cm}$ and $E I=0.05 \mathrm{~g} /\left(\mathrm{cm} \mathrm{s}^{2}\right)$, is immersed in the fluid, clamped at the mid-point $x=0$ bottom of the channel $\Gamma_{\text {down }}$. The beam is initially straight and vertical, and the fluid is initially at rest. We consider a time-dependent FSI problem which is driven by the time-dependent inlet velocity data: a time-dependent Poiseuille velocity profile, with maximum velocity

$$
U(t)=\frac{1}{4}\left(1-\cos \left(\frac{\pi}{2} t\right)\right) \mathrm{cm} / \mathrm{s}
$$

is prescribed at $\Gamma_{i n}$, which corresponds to $x=-3$. A homogeneous Neumann condition is enforced at $\Gamma_{\text {out }}$, i.e., at $x=3$. The no-slip condition is imposed on $\Gamma_{d o w n}$, and a symmetry condition is imposed on $\Gamma_{u p}$. See Fig. 10. The Strouhal number for this problem is 0.5 . The inlet boundary condition and the structural parameters were chosen to generate a "moderate"-amplitude oscillatory motion of the beam around its initial configuration. Fig. 10 shows two snapshots of the velocity magnitude together with the beam position at the time of maximum deflection.

To show that both DN and RN algorithms yield numerical results that are very close, we compare the $x$ coordinate of the beam tip over time. The results, shown in Figure 11(a), indicate that the two curves are superimposed. To zoom into the difference between the two results, we plot the absolute value of the difference between the two curves in Fig. 11(b). One can see that the maximum difference over time interval $[0,10] \mathrm{s}$ is $2 \cdot 10^{-3}$, which occurs after a first ALE reparameterization at around $t=4.8$. 


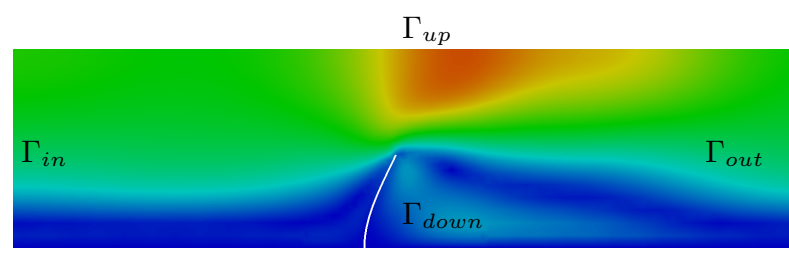

(a) $t=7 \mathrm{~s}$

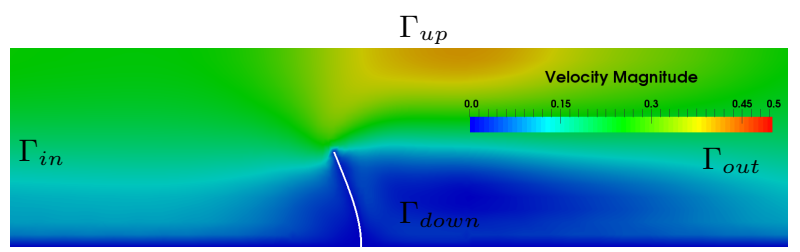

(b) $t=9 \mathrm{~s}$

Figure 10: Velocity magnitude and beam position at time (a) $t=7 \mathrm{~s}$ and (b) $t=9 \mathrm{~s}$.

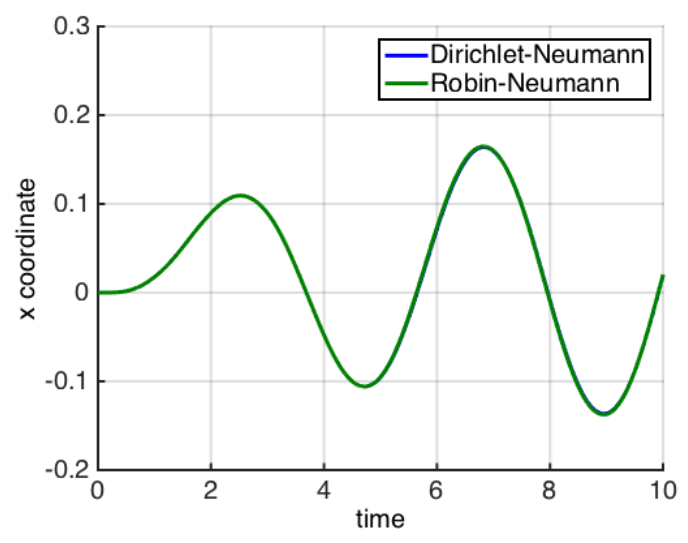

(a) DN and RN

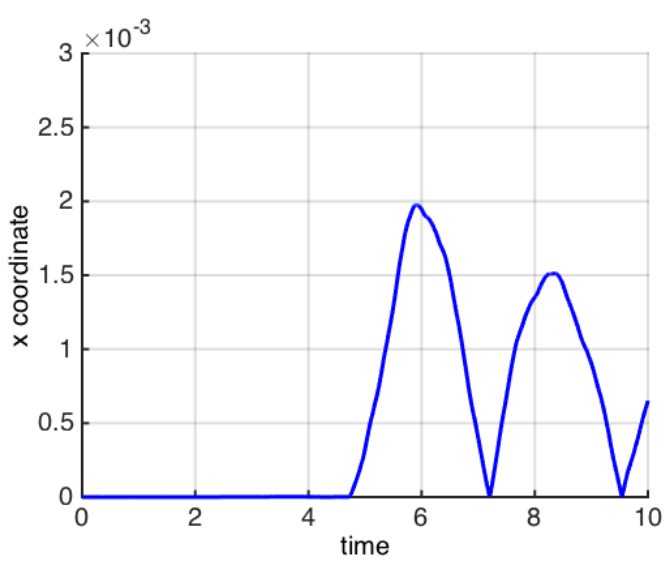

(b) Difference

Figure 11: (a) Comparison of the $x$-component of the beam tip position computed with Dirichlet-Neumann and Robin-Neumann algorithm and (b) difference in absolute value between the curves in (a).

In order to evaluate the performance of the DN and RN algorithms, we let the structure density vary: $\rho_{s}=16,8,4,2,1 \mathrm{~g} / \mathrm{cm}$. The convergence properties of the DN algorithm are known to depend heavily on the added-mass effect, which becomes worse as the structure density gets closer to the density of the fluid, which is $\rho_{f}=1 \mathrm{~g} / \mathrm{cm}^{3}[22]$. Thus, we expect to see an increase in the number of iterations required by the DN method as the ratio $\rho_{s} / \rho_{f}$ approaches one (from above). Indeed, Fig. 12 (a) shows that the number of DN iterations within each time step over time interval $[0,10]$ increases dramatically to 56 as the density ratio $\rho_{s} / \rho_{f}$ approaches 1 . However, when relaxation based on Aitken's acceleration is used, the number of DN iterations decreases significantly. Indeed, Fig. 12 (b) shows that the maximum number of DN iterations with Aitken's acceleration equals 7 when $\rho_{s} / \rho_{f}=1$. 


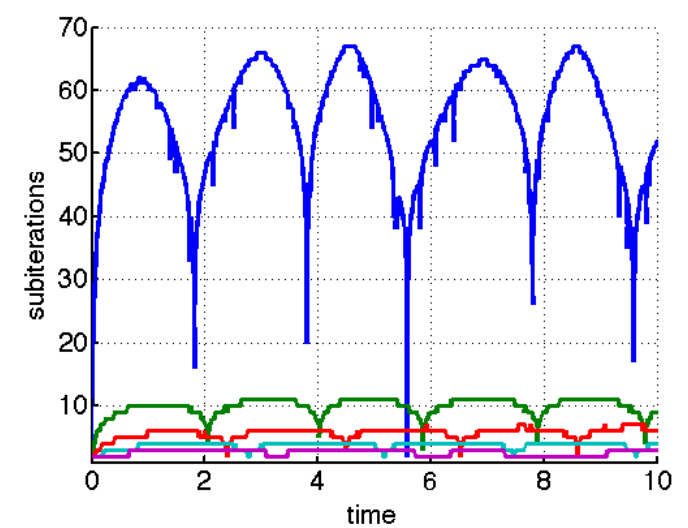

(a) DN with no acceleration

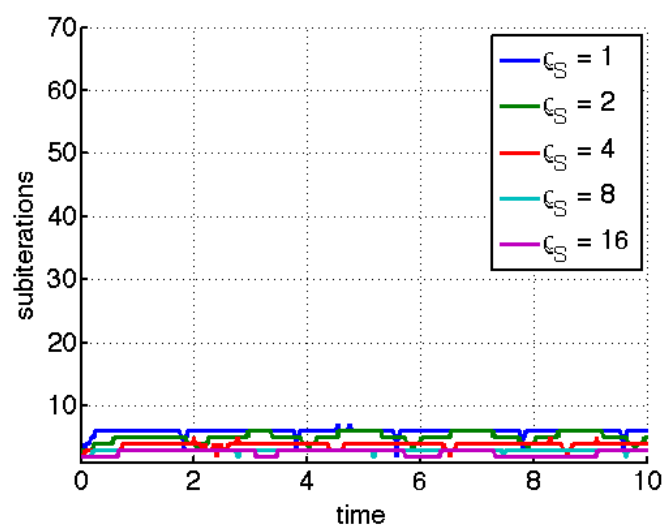

(b) DN with Aitken's acceleration

Figure 12: Number of sub-iterations required by the DN algorithm (a) without acceleration techniques and (b) with Aitken's acceleration technique to converge over time for different for different values of the structure density. The legend in (b) is common to both subfloats.

We next compare the performance of the DN algorithm with Aitken's acceleration and the RN algorithm with no acceleration. Fig. 13(a) shows the same graph as in Fig. 12(b) but on a different scale, and Fig. 13(b) shows the number of iterations required by the $\mathrm{RN}$ algorithm.

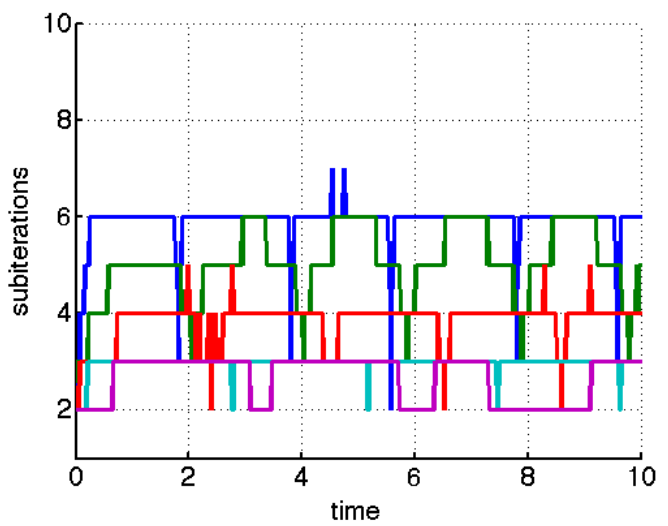

(a) DN with Aitken's acceleration

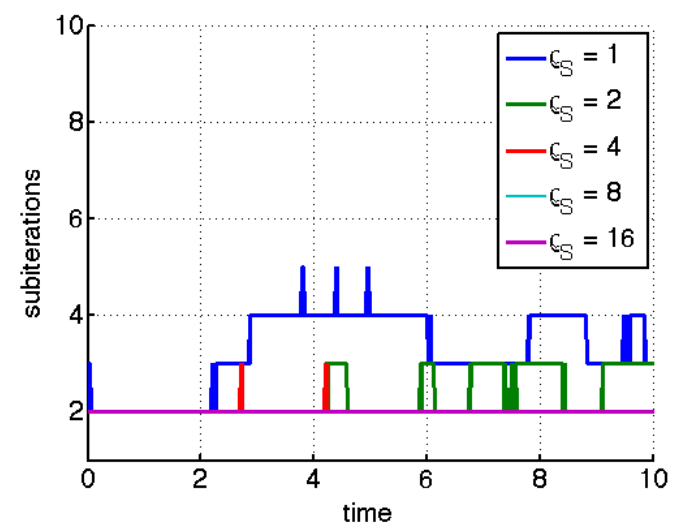

(b) $\mathrm{RN}$

Figure 13: Number of iterations required by (a) the DN algorithm with Aitken's acceleration method and (b) the RN method to converge over time (in s) for different for different values of the structure density. The legend in (b) is common to both subfloats.

We see that for all the structure densities under consideration, the $\mathrm{RN}$ algorithm requires less iterations to converge than the DN method with Aitken's acceleration technique. Moreover, the RN method is quite insensitive to variations in the structure density. In fact, we see in Fig. 13(b) that for $\rho_{s}=16,8,4,2$ $\mathrm{g} / \mathrm{cm}$, the RN algorithm converges in 2 iterations most of the time. Even when $\rho_{s}=1 \mathrm{~g} / \mathrm{cm}$, the number of iterations required by the RN method is at most 5 and only for a very limited time.

\subsubsection{Power exchange at the interface}

We consider the same problem as in Sec. 6.5.1 with structure density $\rho_{s}=5 \mathrm{~g} / \mathrm{cm}$. The goal is to verify how well the power exchanged at the interface is approximated. We first calculate the discrete power 
exchanged at the interface from the fluid side $P^{f}$, defined in (36), computed with two fluid meshes of mesh size $h=0.05 \cdot 2^{j}, j=0,1$. Fig. 14 (a) shows that they are in a pretty good agreement. The occasional jumps in the discrete power that can be seen in Fig. 14 (a) occur whenever the ALE mapping is reparametrized. These jumps are to be expected for dynamically changing meshes, as pointed out in [16, 18].

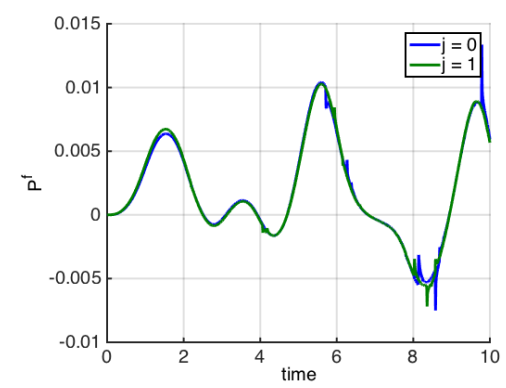

(a) $P^{f}$ : coarse v.s. fine mesh

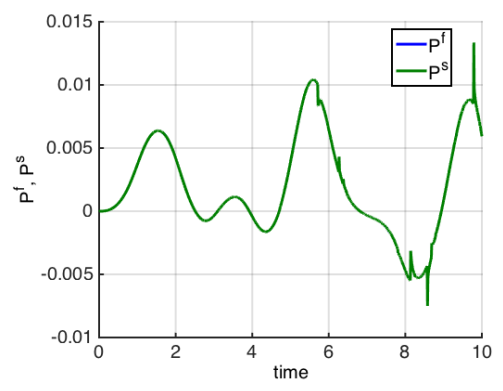

(b) $P^{f}$ and $P^{s}$

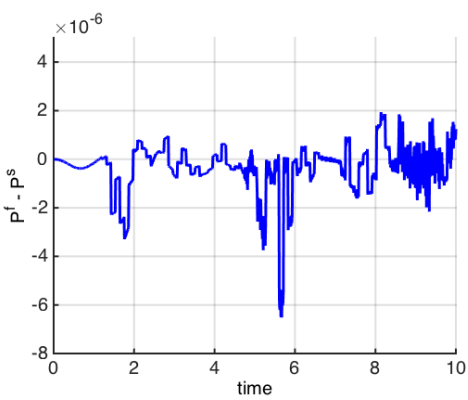

(c) $P^{f}-P^{s}$

Figure 14: Discrete power exchanged at the interface from the fluid side $P^{f}$, defined by (36), computed with two different meshes.

Next, we quantify the unbalance in the power exchange at the interface. As explained in Sec. 5.2, at each time $t^{n+1}$ the powers exchanged at the interface from the fluid side $P^{f, n+1}$ and from the structure side $P^{s, n+1}$ are not necessarily equal. In Fig. 14(b), we plot the powers $P^{f}$ and $P^{s}$ computed with mesh $j=1$ over the time interval under consideration, while in Fig. 14 we show the difference $P^{f}-P^{s}$. One can see in Fig. 14(c )that over a long time interval the difference between the two powers exchanged at the interface is of the order of $10^{-6} \mathrm{~g} \mathrm{~cm} / \mathrm{s}^{3}$. This corresponds to $0.01 \%$ of the power value, which is of the order of $10^{-2} \mathrm{~g}$ $\mathrm{cm} / \mathrm{s}^{3}$, as shown in Fig. 14(b). Such a small difference between $P^{f}$ and $P^{s}$ does not endanger stability.

\subsection{A valve near a contact (regurgitant valve)}

We consider the same fluid domain as in the previous two sections $\Omega_{f}=[-3,3] \times[-0.5,0.5]$, but a longer immersed beam: we set the beam length to be $L=0.95 \mathrm{~cm}$, just short of touching the top boundary $\Gamma_{u p}$. See Fig. 15(a). All the physical and discretization parameters are chosen as in Sec. 6.5.1. The initial beam configuration is vertical, as shown in Fig. 15(a), and the initial fluid velocity is zero.

We study a time-dependent FSI problem in which the fluid flow is driven by the difference in the normal stress prescribed the inlet and outlet, $\Gamma_{i n}$ and $\Gamma_{\text {out }}$. At $\Gamma_{i n}$ the time-dependent normal stress is given by a time-periodic function with period $10 \mathrm{~s}$, where during each period the normal stress is given by the step function:

$$
\boldsymbol{\sigma n}(t, \boldsymbol{x})=\left\{\begin{array}{ll}
{[1.5,0]^{T}} & \text { if } 0 \leq \tilde{t} \leq 3, \\
{[0,0]^{T}} & \text { if } 3<\tilde{t}<10
\end{array} \quad \text { for } \quad \boldsymbol{x} \in \Gamma_{i n}\right.
$$

where $\tilde{t} \in[0,10)$ is $\tilde{t}=\bmod (t, 10)$. Therefore, a constant, normal stress in the horizontal direction is applied for the first $3 \mathrm{~s}$, when the stress is released and set to zero. This is repeated six times until $t=60 \mathrm{~s}$. At $\Gamma_{\text {out }}$, the normal stress is prescribed to be zero. The no-slip condition is imposed on $\Gamma_{d o w n}$, and a symmetry condition is imposed on $\Gamma_{u p}$.

Due to the periodic forcing of the fluid, the induced beam movement will be periodic: the beam is pushed to the right for $0 \leq \tilde{t} \leq 3$, and then bends backward due to elastic forces for $3<\tilde{t}<10$. This motion is repeated six times until $t$ reaches $60 \mathrm{~s}$. See Figure 15. Because of the symmetry condition imposed on the upper wall, this test corresponds to simulating a $1 \mathrm{D}$ valve which is just short of closing, i.e., near a contact. We can see in Figure 15 that there is only one element between the tip of the valve and $\Gamma_{u p}$.

This scenario is typically associated with various problems. In particular, due to the fact that the beam tip is very close to the upper boundary of the fluid domain, the standard ALE method breaks down because 


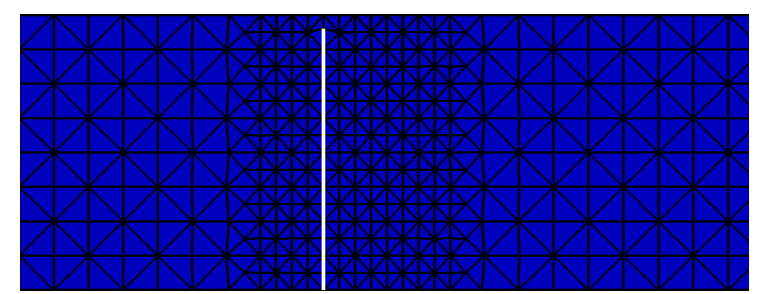

(a) $t=0 \mathrm{~s}$

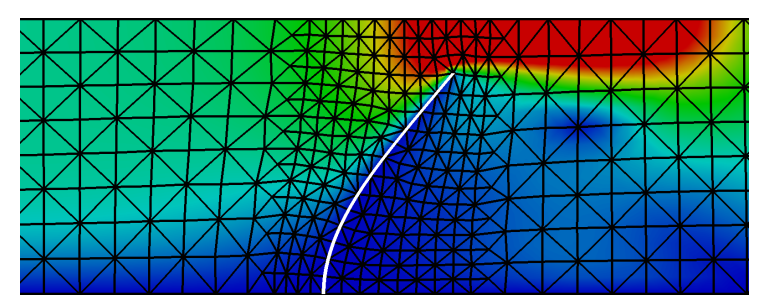

(c) $t=3 \mathrm{~s}$

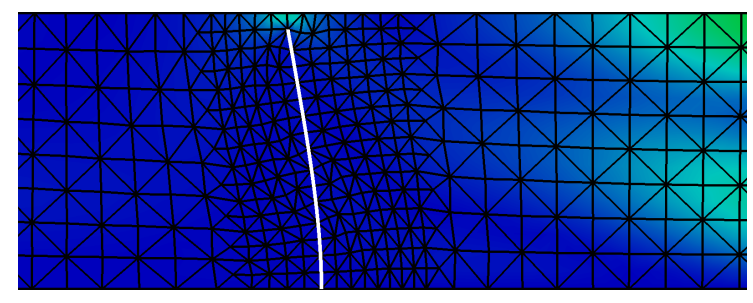

(e) $t=8 \mathrm{~s}$

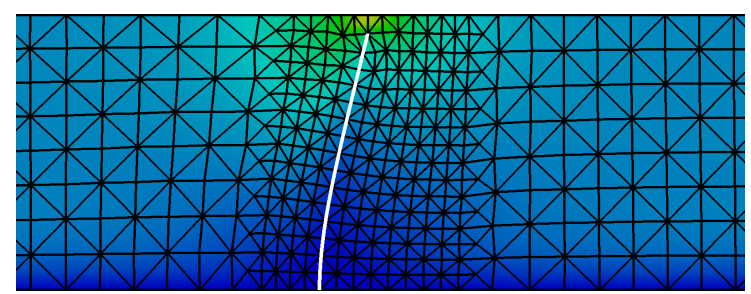

(b) $t=1 \mathrm{~s}$

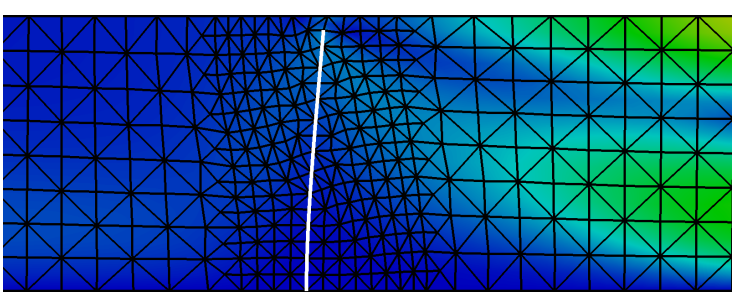

(d) $t=6 \mathrm{~s}$

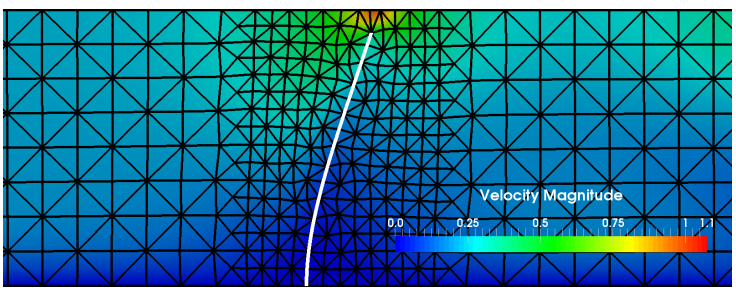

(f) $t=11 \mathrm{~s}$

Figure 15: Beam position and velocity magnitude together with mesh deformation at (a) $t=0 \mathrm{~s}$, (b) $t=1 \mathrm{~s}$, (c) $t=3 \mathrm{~s}$, (d) $t=6 \mathrm{~s}$, (e) $t=8 \mathrm{~s}$, (f) $t=11 \mathrm{~s}$. 
the mesh associated with this simulation gets quickly severely distorted and breaks down before a single cycle of valve motion is completed. The Extended ALE Method, however, does not suffer from this problem. Indeed, Fig. 15 shows that the mesh quality remains good, and that completing a cycle of a near-closing (regurgitant) valve is not a problem.

To show that this method does not induce spurious energy or instabilities over time, we let this simulation run for 6 cycles. Figure 16 shows the $x$ and $y$ coordinates of the beam tip position over time. The movement of the beam tip exhibits perfect monochromatic behavior, showing that our method does not introduce spurious energy over time and that no instabilities arise over a rather long time interval. From Fig. 16(b), we also see that the inextensibility constraint is never violated. In fact, the beam is clamped at $(0,-0.5)$ $\mathrm{cm}$ and its $y$ coordinate never exceeds $0.45 \mathrm{~cm}$, the beam being $0.95 \mathrm{~cm}$ long.



(a) $x$-coordinate

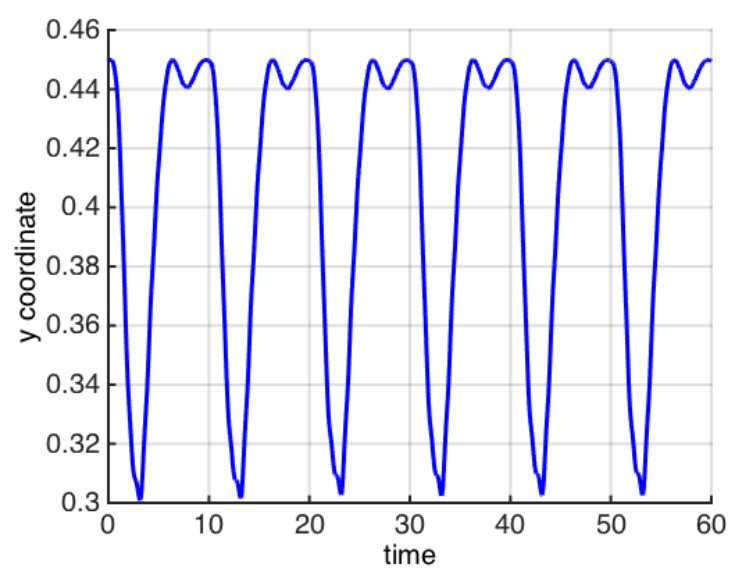

(b) $y$-coordinate

Figure 16: (a) $x$-coordinate and (b) $y$-coordinate of beam tip position (in $\mathrm{cm}$ ) over time (in s).

This example shows that the Extended ALE Method deals well with structure motion near a contact, and that it does not introduce any spurious energy or instabilities over time.

\section{Conclusions}

Standard ALE methods for the simulation of fluid-structure interaction problems fail when the structural displacement is large. In this paper, we proposed an Extended ALE Method to overcome this limitation without remeshing. Our method relies on a variational mesh optimization technique with an additional constraint to enforce the alignment of the structure interface with edges of the resulting triangulation.

The performance of the Extended ALE Method was evaluated on a series of test examples involving FSI problems with two-way coupling, and large displacements. We considered two partitioned algorithms: the classical Dirichlet-Neumann method with and without Aitken's acceleration, and the Robin-Neumann method. Each partitioned algorithm was combined with the Extended ALE Method to solve the FSI problems. Because the Extended ALE Method provides a fluid mesh aligned with the interface, non-trivial boundary conditions such as the Robin boundary conditions can be easily implemented. We showed that in problems where the structure density is close to the fluid density, the Robin-Neumann method outperforms the Dirichlet-Neumann algorithms both with and without Aitken's acceleration. In fact, in balloon-like problems, the Dirichlet-Neumann algorithms fail, while the Robin-Neumann algorithm combined with the Extended ALE Method performs well, and appears insensitive to the added mass effect even when $\rho_{s} / \rho_{f} \approx 1$.

The test problems presented in this manuscript were carefully chosen to study the performance of our approach in FSI problems with large displacements. They involve various scenarios of an elastic, possibly inextensible beam interacting with an incompressible, viscous fluid in $2 \mathrm{D}$. The examples clearly show that the 
Extended ALE Method is in excellent agreement with the results obtained using a standard ALE method when structural displacement is small. For large displacements and in problems with structures near a contact, when standard ALE methods fail, we showed that the quality of the mesh given by the Extended ALE Method remains high, thereby allowing full computer simulations of this class of problems.

Due to the simplicity and their instructive nature, the test problems presented in this manuscript can be used as benchmark problems in the development of numerical tools for the computer simulation of FSI problems involving immersed structures with large displacements, and in FSI problems involving structures near a contact.

Preliminary results using the proposed mesh optimization techniques for 3D fluid/rigid particle systems have been obtained and will be presented in a forthcoming paper. Future developments of this work include the extension to more complex structure models and 3D biomedical applications, e.g. blood interacting with heart valves.

\section{Acknowledgments}

The research presented in this work was carried out during Basting's visits at University of Houston, supported by National Science Foundation (NSF) grant DMS-1318763 (Canic) and Cullen Chair funds. Additionally, this research has been supported in part by the NSF under grants DMS-1311709, DMS-1613757 (Canic), DMS-1262385 and by DMS-1109189 (Canic and Quaini).

[1] S.V. Apte, M. Martin, and N.A. Patankar. A numerical method for fully resolved simulation (frs) of rigid particleflow interactions in complex flows. Journal of Computational Physics, 228(8):2712 - 2738, 2009.

[2] M. Astorino, J.-F. Gerbeau, O. Pantz, and K.-F. Traoré. Fluid-structure interaction and multi-body contact: Application to aortic valves. Computer Methods in Applied Mechanics and Engineering, 198(45-46):3603 - 3612, 2009.

[3] F.P.T. Baaijens. A fictitious domain/mortar element method for fluid-structure interaction. Int. J. Numer. Meth. Fl., 35(7):743-761, 2001

[4] S. Badia, F. Nobile, and C. Vergara. Fluid-structure partitioned procedures based on Robin transmission conditions. Journal of Computational Physics, 227:7027-7051, 2008.

[5] J. Baiges and R. Codina. The fixed-mesh ALE approach applied to solid mechanics and fluid-structure interaction problems. International journal for numerical methods in engineering, 81(12):1529-1557, 2010.

[6] J.W. Banks, W.D. Henshaw, and Schwendeman. Deforming composite grids for solving fluid structure problems. Journal of Computational Physics, 231(9):3518-3547, 2012.

[7] J.W. Banks, W.D. Henshaw, and Schwendeman. An added-mass partitioned algorithm for fluid-structure interactions of compressible fluids and nonlinear solids. Journal of Computational Physics, 305:1037-1064, 2016.

[8] J.W. Banks, W.D. Henshaw, and D.W. Schwendeman. An analysis of a new stable partitioned algorithm for FSI problems. part ii: Incompressible flow and structural shells. Journal of Computational Physics, 268:399 - 416, 2014.

[9] E. Bänsch, J. Paul, and A. Schmidt. An ALE finite element method for a coupled Stefan problem and Navier-Stokes equations with free capillary surface. International Journal for Numerical Methods in Fluids, 2012.

[10] S. Basting and R. Prignitz. An interface-fitted subspace projection method for finite element simulations of particulate flows. Computer Methods in Applied Mechanics and Engineering, 267(0):133 - 149, 2013.

[11] S. Basting, A. Quaini, R. Glowinski, and S. Canic. Comparison of time discretization schemes to simulate the motion of an inextensible beam. In Numerical Mathematics and Advanced Applications - ENUMATH 2013. In Lecture Notes in Computational Science and Engineering, volume 103, pages 175-183, 2015.

[12] S. Basting and M. Weismann. A hybrid level set/front tracking finite element approach for fluid-structure interaction and two-phase flow applications. Journal of Computational Physics, 255:228 - 244, 2013.

[13] S. Basting and M. Weismann. A hybrid level set/front tracking approach for finite element simulations of two-phase flows. Journal of Computational and Applied Mathematics, 270:471-483, 2014.

[14] K. Bäumler and E. Bänsch. A subspace projection method for the implementation of interface conditions in a single-drop flow problem. Journal of Computational Physics, 252:438 - 457, 2013.

[15] B. Bejanov, J.L. Guermond, and P.D. Minev. A grid-alignment finite element technique for incompressible multicomponent flows. Journal of Computational Physics, 227(13):6473 - 6489, 2008.

[16] M. Besier and W. Wollner. On the pressure approximation in nonstationary incompressible flow simulations on dynamically varying spatial meshes. International Journal for Numerical Methods in Fluids, 69(6):1045-1064, 2012.

[17] J.-F. Bourgat, M. Dumay, and R. Glowinski. Large displacement calculations of inexstensible pipelines by finite element and nonlinear programming methods. SIAM J. Sci. Stat. Comput., 1:34-81, 1980.

[18] A. Brenner, E. Bänsch, and M. Bause. A priori error analysis for finite element approximations of the Stokes problem on dynamic meshes. IMA Journal of Numerical Analysis, page drt001, 2013.

[19] M. Bukac, S. Canic, R. Glowinski, J. Tambaca, and A. Quaini. Fluid-structure interaction in blood flow capturing non-zero longitudinal structure displacement. Journal of Computational Physics, 235:515-541, 2013.

[20] E. Burman and M.A. Fernández. Stabilization of explicit coupling in fluid-structure interaction involving fluid incompressibility. Computer Methods in Applied Mechanics and Engineering, 198:766-784, 2009. 
[21] A.T. Cate, J.J. Derksen, L.M. Portella, and H.E.A. Van Den Akker. Fully resolved simulations of colliding monodisperse spheres in forced isotropic turbulence. Journal of Fluid Mechanics, 519:233-271, 112004.

[22] P. Causin, J.F. Gerbeau, and F. Nobile. Added-mass effect in the design of partitioned algorithms for fluid-structure problems. Comput. Methods Appl. Mech. Engrg, 194(42-44):4506-4527, 2005.

[23] K. C. Chang, L. Sanchez, and P. Rabinowitz. Nontrivial periodic solutions of a nonlinear beam equation. Mathematical Methods in the Applied Sciences, 4(1):194-205, 1982.

[24] J. Chen and D. O'Regan. The periodic boundary value problem for semilinear elastic beam equations: the resonance case. Computers \& Mathematics with Applications, 53(8):1284-1292, 2007.

[25] R. Codina, G. Houzeaux, H. Coppola-Owen, and J. Baiges. The fixed-mesh ALE approach for the numerical approximation of flows in moving domains. Journal of Computational Physics, 228(5):1591-1611, 2009.

[26] M. Coquerelle and G.-H. Cottet. A vortex level set method for the two-way coupling of an incompressible fluid with colliding rigid bodies. Journal of Computational Physics, 227(21):9121 - 9137, 2008.

[27] G.H. Cottet, E. Maitre, and T. Milcent. Eulerian formulation and level set models for incompressible fluid-structure interaction. Esaim. Math. Model. Numer. Anal., 42:471-492, 2008.

[28] J. Donea, S. Giuliani, and J.P. Halleux. An Arbitrary Lagrangian-Eulerian finite element method for transient dynamic fluid-structure interactions. Computer Methods in Applied Mechanics and Engineering, 33(1-3):689 - $723,1982$.

[29] N. Diniz dos Santos, J.-F. Gerbeau, and J.-F. Bourgat. A partitioned fluid-structure algorithm for elastic thin valves with contact. Comput. Methods Appl. Mech. Engrg, 197:1750-1761, 2008.

[30] J. Dukowicz and J. Kodis. Accurate conservative remapping (rezoning) for Arbitrary Lagrangian-Eulerian computations. SIAM Journal on Scientific and Statistical Computing, 8(3):305-321, 1987.

[31] T. Dunne. An Eulerian approach to fluid-structure interaction and goal-oriented mesh adaptation. International journal for numerical methods in fluids, 51(9-10):1017-1039, 2006.

[32] H. Fang, Z. Wang, Z. Lin, and M. Liu. Lattice Boltzmann method for simulating the viscous flow in large distensible blood vessels. Phys. Rev. E., 65(5):051925-1-051925-12, 2002.

[33] C. Farhat, M. Lesoinne, and P. Le Tallec. Load and motion transfer algorithms for fluid/structure interaction problems with non-matching discrete interfaces: Momentum and energy conservation, optimal discretization and application to aeroelasticity. Computer Methods in Applied Mechanics and Engineering, 157(12):95 - 114, 1998.

[34] Z.G. Feng and E.E. Michaelides. The immersed boundary-lattice Boltzmann method for solving fluid-particles interaction problems. J. Comp. Phys., 195(2):602-628, 2004

[35] L. Formaggia, A. Quarteroni, and A. Veneziani. Cardiovascular Mathematics, volume 1 of Modeling, Simulation and Applications. Springer, 2009.

[36] M. Fortin and R. Glowinski. Augmented Lagrangian Methods: Application to the Numerical Solution of Boundary Value Problem. North-Holland, Amsterdam, 1983.

[37] L. A. Freitag and P. M. Knupp. Tetrahedral element shape optimization via the jacobian determinant and condition number. In $I M R$, pages 247-258, 1999.

[38] L. A. Freitag and P. M. Knupp. Tetrahedral mesh improvement via optimization of the element condition number. International Journal for Numerical Methods in Engineering, 53(6):1377-1391, 2002.

[39] T.-P. Fries and T. Belytschko. The extended/generalized finite element method: an overview of the method and its applications. International Journal for Numerical Methods in Engineering, 84(3):253-304, 2010.

[40] E. S. Gawlik, H. Kabaria, and A. J. Lew. High-order methods for low Reynolds number flows around moving obstacles based on universal meshes. International Journal for Numerical Methods in Engineering, 2015.

[41] E. S. Gawlik and A. J. Lew. High-order finite element methods for moving boundary problems with prescribed boundary evolution. Computer Methods in Applied Mechanics and Engineering, 278:314-346, 2014.

[42] F. Gibou and C. Min. Efficient symmetric positive definite second-order accurate monolithic solver for fluid/solid interactions. Journal of Computational Physics, 231(8):3246 - 3263, 2012.

[43] R. Glowinski and M. Holmstrom. Constrained motion problems with applications by nonlinear programming methods. Surv. Math. Ind., 5:75-108, 1995.

[44] R. Glowinski, T.-W. Pan, T.I. Hesla, and D.D. Joseph. A distributed Lagrange multiplier/fictitious domain method for particulate flows. International Journal of Multiphase Flow, 25(5):755 - 794, 1999.

[45] R. Glowinski, T.W. Pan, and J. Périaux. A fictitious domain method for external incompressible viscous flow modelled by Navier-Stokes equations. Methods Appl. Mech. Engrg., 111:133-148, 1994.

[46] R. Glowinski and P. Le Tallec. Augmented Lagrangian and Operator-Splitting Methods in Nonlinear Mechanics. SIAM, Philadelphia, 1988.

[47] S. Gross and A. Reusken. Numerical methods for two-phase incompressible flows, volume 40. Springer Science \& Business Media, 2011.

[48] G. Guidoboni, R. Glowinski, N. Cavallini, and S. Canic. Stable loosely-coupled-type algorithm for fluid-structure interaction in blood flow. Journal of Computational Physics, 228(18):6916-6937, 2009.

[49] J. De Hart, F.P.T. Baaijens, G.W.M. Peters, and P.J.G. Schreurs. A computational fluid-structure interaction analysis of a fiber-reinforced stentless aortic valve. Journal of Biomechanics, 36(5):699 - 712, 2003.

[50] J. De Hart, G.W.M. Peters, P.J.G. Schreurs, and F.P.T. Baaijens. A three-dimensional computational analysis of fluidstructure interaction in the aortic valve. Journal of Biomechanics, 36(1):103 - 112, 2003.

[51] C.W Hirt, A.A Amsden, and J.L Cook. An arbitrary lagrangian-eulerian computing method for all flow speeds. Journal of Computational Physics, 14(3):227 - 253, 1974.

[52] H. Hu, N.A. Patankar, and M.Y. Zhu. Direct numerical simulations of fluid-solid systems using the Arbitrary LagrangianEulerian technique. Journal of Computational Physics, 169(2):427 - 462, 2001 
[53] T. J. R. Hughes, W. Liu, and T. K. Zimmermann. Lagrangian-Eulerian finite element formulation for incompressible viscous flows. Computer Methods in Applied Mechanics and Engineering, 29(3):329 - 349, 1981.

[54] M. Krafczyk, M. Cerrolaza, M. Schulz, and E. Rank. Analysis of 3D transient blood flow passing through an artificial aortic valve by Lattice-Boltzmann methods. J. Biomech., 31(5):453-462, 1998.

[55] U. Küttler and W.A. Wall. Fixed-point fluid-structure interaction solvers with dynamic relaxation. Computational Mechanics, 43(1):61-72, 2008.

[56] A. Kuzmin, M. Luisier, and O. Schenk. Fast methods for computing selected elements of the Greens function in massively parallel nanoelectronic device simulations. In F. Wolf, B. Mohr, and D. Mey, editors, Euro-Par 2013 Parallel Processing, volume 8097 of Lecture Notes in Computer Science, pages 533-544. Springer Berlin Heidelberg, 2013.

[57] L. Li, W.D. Henshaw, J.W. Banks, D.W. Schwendeman, and A. Main. A stable partitioned fsi algorithm for incompressible flow and deforming beams. Journal of Computational Physics, 312:272-306, 2016.

[58] R. Loubere, P.-H. Maire, M. Shashkov, J. Breil, and S. Galera. Reale: A reconnection-based arbitrary-Lagrangian-Eulerian method. Journal of Computational Physics, 229(12):4724 - 4761, 2010.

[59] L.G. Margolin and M. Shashkov. Second-order sign-preserving conservative interpolation (remapping) on general grids. Journal of Computational Physics, 184(1):266 - 298, 2003.

[60] D.P. Mok and W.A. Wall. Partitioned analysis schemes for transient interaction of incompressible flows and nonlinear flexible structures. In Trends in computational structural mechanics (W.A. Wall, K.U. Bletzinger and K. Schweizerhof, Eds.), CIMNE, Barcelona, Spain, 2001.

[61] F. Nobile. Numerical Approximation of Fluid-Structure Interaction Problems with Application to Haemodynamics. PhD thesis, École Polytechnique Fédérale de Lausanne, 2001.

[62] F. Nobile and C. Vergara. An effective fluid-structure interaction formulation for vascular dynamics by generalized Robin conditions. SIAM J. Sci. Comp., 30(2):731-763, 2008.

[63] WF Noh. CEL: A time-dependent, two-space-dimensional, coupled Eulerian-Lagrange code. Technical report, Lawrence Radiation Lab., Univ. of California, Livermore, 1963.

[64] C. S. Peskin. Numerical analysis of blood flow in the heart. Journal of Computational Physics, 25(3):220 - 252, 1977.

[65] C. S. Peskin. The immersed boundary method. Acta Numer., 11:479-517, 2002.

[66] C.S. Peskin and D.M. McQueen. A three-dimensional computational method for blood flow in the heart i. Immersed elastic fibers in a viscous incompressible fluid. Journal of Computational Physics, 81(2):372 - 405, 1989.

67] R. Prignitz and E. Bänsch. Particulate flows with the subspace projection method. Journal of Computational Physics, 260(0):249-272, 2014.

[68] A. Quarteroni and A. Valli. Numerical Approximation of Partial Differential Equations. Springer-Verlag, 1994.

[69] A. Quarteroni and A. Valli. Domain Decomposition Methods for Partial Differential Equations. Oxford Science Publications, 1999.

[70] R. Rangarajan and A. J. Lew. Analysis of a method to parameterize planar curves immersed in triangulations. SIAM Journal on Numerical Analysis, 51(3):1392-1420, 2013.

[71] T. Richter and T. Wick. Finite elements for fluid-structure interaction in ALE and fully Eulerian coordinates. Computer Methods in Applied Mechanics and Engineering, 199(41):2633-2642, 2010.

[72] A. Robinson-Mosher, T. Shinar, J. Gretarsson, J. Su, and R. Fedkiw. Two-way coupling of fluids to rigid and deformable solids and shells. ACM Transactions on Graphics (TOG) - Proceedings of ACM SIGGRAPH 2008, 27(3):article 46, 2008.

[73] M. Rumpf. A variational approach to optimal meshes. Numerische Mathematik, 72:523-540, 1996.

[74] O. Schenk, M. Bollhöfer, and R. A. Römer. On large-scale diagonalization techniques for the Anderson model of localization. SIAM Rev., 50(1):91-112, February 2008.

[75] O. Schenk, A. Wchter, and M. Hagemann. Matching-based preprocessing algorithms to the solution of saddle-point problems in large-scale nonconvex interior-point optimization. Computational Optimization and Applications, 36(2-3):321$341,2007$.

[76] P. Le Tallec and J. Mouro. Fluid-structure interaction with large structural displacements. Comput. Methods Appl. Mech. Engrg, 190:3039-3067, 2001.

[77] R. van Loon, P. D. Anderson, J. de Hart, and F. P. T. Baaijens. A combined fictitious domain/adaptive meshing method for fluid-structure interaction in heart valves. International Journal for Numerical Methods in Fluids, 46(5):533-544, 2004.

[78] R. van Loon, P.D. Anderson, F.P.T. Baaijens, and F.N. van de Vosse. A three-dimensional fluid-structure interaction method for heart valve modelling. Comptes Rendus Mécanique, 333(12):856 - 866, 2005.

[79] X. Wang and W. Liu. Extended immersed boundary method using FEM and RKPM. Methods Appl. Mech. Engrg., 193:1305 - 1321, 2004

[80] M. Weismann. The hybrid level-set front-tracking approach. Master's thesis, Friedrich-Alexander University ErlangenNuremberg, 2012.

[81] T. Wick. Flapping and contact FSI computations with the fluid-solid interface-tracking/interface-capturing technique and mesh adaptivity. Computational Mechanics, 53(1):29-43, 2014.

[82] L. Zhang, A. Gerstenberger, X. Wang, and W.K. Liu. Immersed finite element method. Methods Appl. Mech. Engrg., 193:2051 - 2067, 2004. 